DOI: $10.52119 /$ LPHS . 2019.52.58.001

\title{
ЛОГИКА СЕГОДНЯ
}

\author{
Владимир Попов
}

К ПРОБЛЕМЕ РАСШИРЕНИЯ МАТРИЧНОЙ СЕМАНТИКИ, АДЕКВАТНОЙ КЛАССИЧЕСКОЙ ИМПЛИКАТИВНОЙ ЛОГИКЕ, ДО МАТРИЧНОЙ СЕМАНТИКИ, АДЕКВАТНОЙ КЛАССИЧЕСКОЙ
ИМПЛИКАТИВНО-НЕГАТИВНОЙ ЛОГИКЕ²

Аннотация. Предлагаемая работа выполнена в рамках исследований проблемы расширения семантики, адекватной собственному фрагменту логики, до семантики, адекватной этой логике. Автором обнаружена трехзначная логическая матрица $M(1,0,0,1 / 2)$ с единственным выделенным значением, адекватная классической импликативной логике $C l_{\supset}$ и обладающая следующим свойством: не существует такой унарной операции $f$, чтоупорядоченная пара $\langle M(1,0,0,1 / 2), f\rangle$ является логической матрицей, адекватной классической импликативно-негативной логике $C l_{\supset \neg}$. В данной статье описана упомянутая выше логическая матрица $M(1,0,0,1 / 2)$, определено понятие регулярной $L_{\supset \neg^{-}}$ логики (согласно этому определению логика $C l_{\supset}$ служит примером регулярной $L_{\supset \neg-}$ логики) и доказано следующее: длявсякойунарнойоперации $f$ на носителе логической матрицы $M(1,0,0,1 / 2)$ упорядоченная пара $\langle M(1,0,0,1 / 2), f\rangle$ есть такая логическая матрица, что множество всех общезначимых в $\langle M(1,0,0,1 / 2), f\rangle$ формул не является

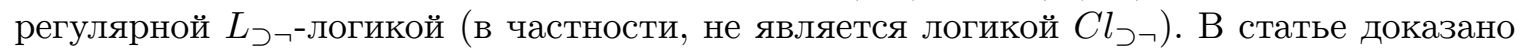
также, что для всякого целого положительного числа $n$ существует такая $n+3$-значная логическая матрица $K$ с единственным выделенным значением, адекватная классической импликативной логике и удовлетворяющая условию: длявсякойунарнойоперации $f$ на носителе этой логической матрицы упорядоченная пара $\langle K, f\rangle$ есть такая логическая матрица, что множество всех общезначимых в $\langle K, f\rangle$ формул не является логикой $C l_{\supset \neg}$.

Ключевые слова: трехзначная логическая матрица с одним выделенным значением,

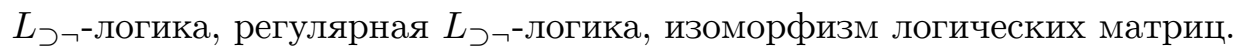

\footnotetext{
${ }^{1}$ Попов Владимир Михайлович - к. филос. н., доцент кафедры логики философского факультета МГУ имени М. В. Ломоносова.

Vladimir Popov, PhD, associate professor, Dept. of Logic, Lomonosov Moscow State University. pphiloslog@mail.ru

${ }^{2}$ Работа выполнена при поддержке РФФИ, проект № 19-011-00536А.
} 
Владимир Попов. К проблеме расширения матричной семантики...

Vladimir Popov

ON THE PROBLEM OF EXPANSION OF MATRIX SEMANTICS ADEQUATE TO CLASSICAL IMPLICATIVE LOGIC TO MATRIX SEMANTICS ADEQUATE TO CLASSICAL IMPLICATIVE-NEGATIVE LOGIC

Abstract. The present work is carried out in the framework of studies of the problem of expansion of a semantics adequate to a proper fragment of a logic to a semantics adequate to this logic. The author has found a three-valued logical matrix $M(1,0,0,1 / 2)$ with one designated value, adequate to the classical implicative logic $C l_{\supset}$ and having the following property: there is no unary operation $f$, such that the ordered pair $\langle M(1,0,0,1 / 2), f\rangle$ is a logical matrix adequate to the classical implicative-negative logic $C l_{\supset \neg}$. This article describes the above mentioned logical matrix $M(1,0,0,1 / 2)$, defines the concept of regular $L_{\supset \neg-\operatorname{logic}}$ (according to this definition the logic $C l_{\supset \neg}$ is an example of a regular $L_{\supset \neg}$-logic) and proves the following: for all unary operations $f$ on the carrier of a logical matrix $M(1,0,0,1 / 2)$, an ordered pair $\langle M(1,0,0,1 / 2), f\rangle$ is a logical matrix such that the set of all valid in $\langle M(1,0,0,1 / 2), f\rangle$ formulas is not a regular $L_{\supset \neg}$-logic (in particular, is not the logic $\left.C l_{\supset \neg}\right)$. The article also proves that for any positive integer $n$ there exists a $n+3$-valued logical matrix $K$ with one designated value, adequate to the classical implicative logic and satisfying the condition: for any unary operation $f$ on the carrier of this logical matrix, the ordered pair $\langle K, f\rangle$ is a logical matrix such that the set of all valid in $\langle K, f\rangle$ formulas is not the logic $C l_{\supset \neg}$. The conclusion of the article contains the following announcement: every three-valued logical matrix $K$ which has a single designated value, is adequate to the logic $C l \supset$ and for which there is no unary operation $f$ such that $\langle K, f\rangle$ is a logical matrix

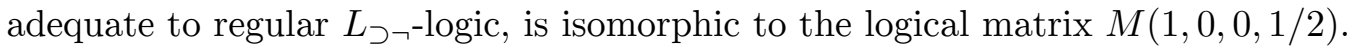

Keywords: three-valued logical matrix with one designated value, $L_{\supset}$-logic, $L_{\supset \neg}$-logic, regular $L_{\supset \neg}$-logic, isomorphism of logical matrices.

Нам потребуются два стандартно определяемых пропозициональных языка $L_{\supset}$ и $L_{\supset \neg}$. Алфавит языка $L_{\supset}$ есть множество $\{\supset),,\left(, p_{1}, p_{2}, p_{3}, \ldots\right\}$ символов, а алфавит языка $L_{\supset\urcorner}$ есть множество $\{\supset, \neg),,\left(, p_{1}, p_{2}, p_{3}, \ldots\right\}$ символов. Здесь $\supset$ есть бинарная логическая связка языка $L_{\supset}$ и языка $L_{\supset\urcorner}, \neg$ есть унарная логическая связка языка $L_{\supset\urcorner}$, ) и ( являются техническими символами языка $L_{\supset}$ и языка $L_{\supset \neg}$, а $p_{1}, p_{2}, p_{3}, \ldots$ являются пропозициональными переменными языка $L_{\supset}$ и языка $L_{\supset \neg}$. Определение $L_{\supset}$-формулы индуктивно: $(1)$ пропозициональная переменная языка $L_{\supset}$ есть $L_{\supset}$-формула, $(2)$ если $A$ есть $L_{\supset}$-формула и $B$ есть $L_{\supset}$-формула, то $(A \supset B)$ есть $L_{\supset}$-формула, $(3)$ ничто другое $L_{\supset}$-формулой не является. Определение $L_{\supset \neg}$-формулы индуктивно и состоит в точности из четырех пунктов, первые два из которых аналогичны первым двум пунктам определения $L_{\supset}$-формулы. Третий пункт определения $L_{\supset \neg}$-формулы: если $A$ есть $L_{\supset \neg}$-формула, то $(\neg A)$ есть $L_{\supset \neg}$-формула. Четвертый пункт определения $L_{\supset\urcorner}$-формулы аналогичен третьему пункту определения $L_{\supset}$-формулы. Называем $L_{\supset}$-логикой множество $L_{\supset}$-формул, замкнутое относительно правила modus ponens в $L_{\supset}$ и относительно правила пропозициональной подстановки в $L_{\supset}$. На-

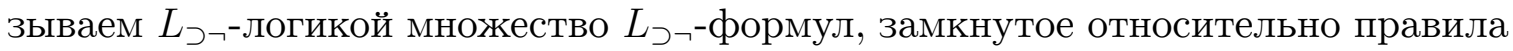


modus ponens в $L_{\supset \neg}$ и относительно правила пропозициональной подстановки в $L_{\supset \neg}$.

Определение 1. Называем $L_{\supset}$-матричей упорядоченную тройку $\langle M, N, g\rangle$, где $M$ есть непустое множество, $N$ есть подмножество множества $M, g$ есть бинарная операция на $M$; при этом $M$ называем носителем $L_{\supset}$-матрицы $\langle M, N, g\rangle, N$ называем выделенным множеством $L_{\supset}$-матрицы $\langle M, N, g\rangle, g$ называем операчией $L_{\supset}$-матрищы $\langle M, N, g\rangle$.

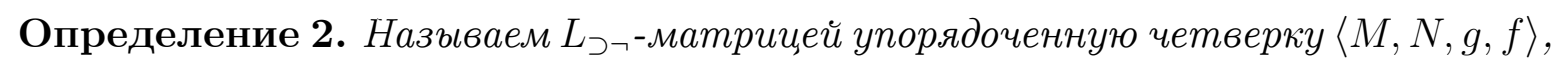
где $M$ есть непустое множество, $N$ есть подмножество множества $M, g$ есть бинарная операция на $M, f$ есть унарная операция на $M$; при этом

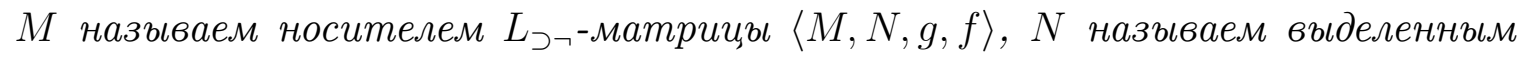

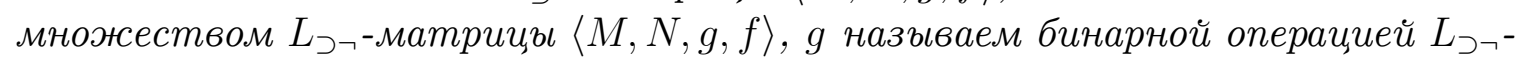

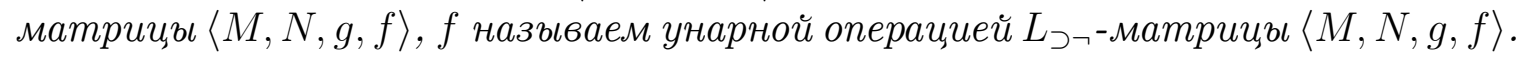

Определение 3. Оценкой языка $L_{\supset}$ в $L_{\supset}$-матрице $K$ называем отображение множества $\left\{p_{1}, p_{2}, p_{3}, \ldots\right\}$ всех пропозиционалъных переменных языка $L_{\supset} в$ носитель $L_{\supset}$-матрицы $K$.

Замечание 1. Можно доказать, что для всякой $L_{\supset}$-матрицъ K существует единственное отображение (обозначаем его через $\varphi_{K}$ ) множества всех упорядоченных пар, каждая из которых имеет вид $\langle A, w\rangle$, где $A$ есть $L_{\supset}$-бормула

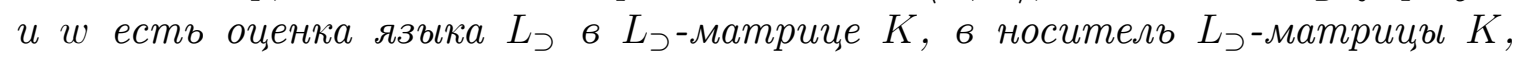
выполняющее следующие два условия: (1) для всякой пропозищиональной пере-

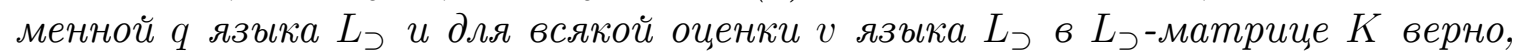
что $\varphi_{K}(\langle q, v\rangle)=v(q)$, (2) для всякой $L_{\supset}$-формуль $A$, для всякой $L_{\supset}$-формульь $B$ и для всякой оценки $v$ языка $L_{\supset}$ в $L_{\supset \text {-матрице } K} \varphi_{K}(\langle(A \supset B), v\rangle)=$ $\left(\varphi_{K}(\langle A, v\rangle) g \varphi_{K}(\langle(B, v\rangle))\right.$, где g есть операция $L_{\supset}$-матрицы $K$.

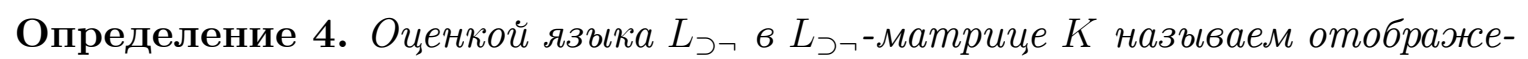
ние множества $p_{1}, p_{2}, p_{3}, \ldots$ всех пропозичиональных переменных языка $L_{\supset\urcorner} 8$

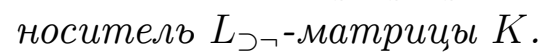

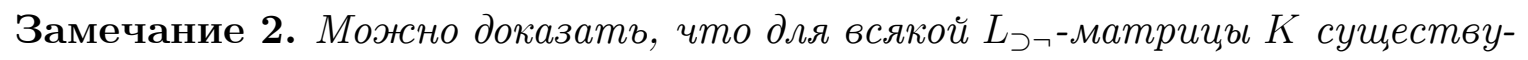
ет единственное отображение (обозначаем его через $\varphi_{K}$ ) множества всех упорядоченных пар, каждая из которых имеет вид $\langle A, w\rangle$, где $A$ есть $L_{\supset\urcorner^{-}}$

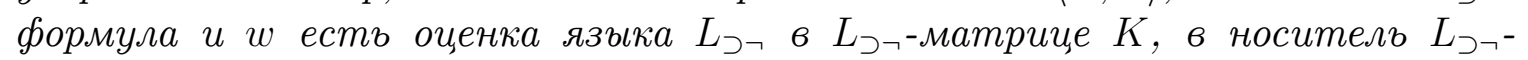
матрищъ K, выполняющее следующие три условия: (1) для всякой пропозициональной переменной $q$ языка $L_{\supset\urcorner}$ и для всякой оценки $v$ языка $L_{\supset\urcorner}$ в

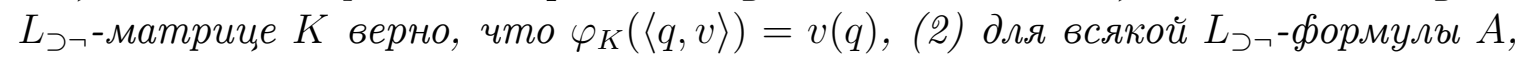

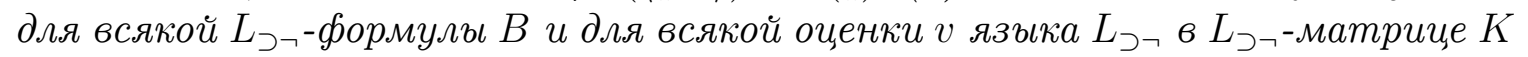
$\varphi_{K}(\langle(A \supset B), v\rangle)=\left(\varphi_{K}(\langle A, v\rangle) g \varphi_{K}(\langle(B, v\rangle))\right.$, где g есть бинарная операция $L_{\supset\urcorner^{-}}$

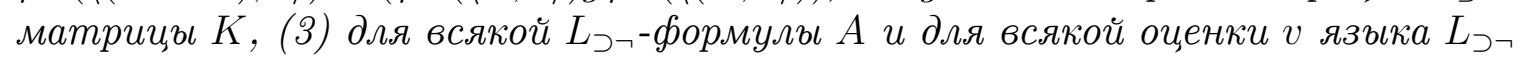

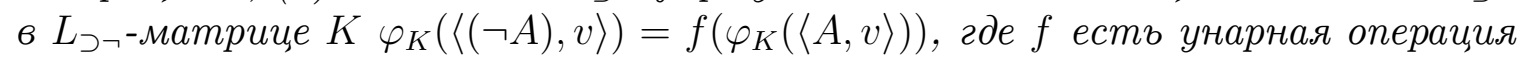

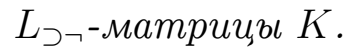


Владимир Попов. К проблеме расширения матричной семантики...

Определение 5. Называем $L_{\supset}$-формулу $A L_{\supset}$-формулой, общезначимой в $L_{\supset}$ матрище $K$, если для всякой оценки $v$ языка $L_{\supset}$ в $L_{\supset}$-матрище $K \varphi_{K}(\langle A, v\rangle)$ принадлежит выделенному множеству $L_{\supset}$-матрицы $K$.

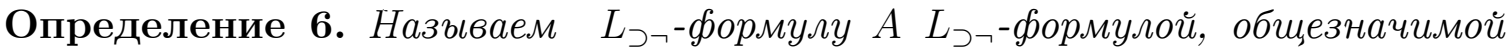

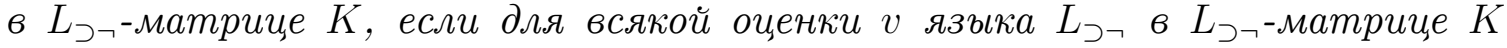
$\varphi_{K}(\langle A, v\rangle)$ принадлежит выделенному множеству $L_{\supset}-$-матрицы $K$.

Определение 7. Называем $L_{\supset \text {-матричу } K} L_{\supset}$-матрицей, адекватной $L_{\supset}$ логике $L$, если для всякой $L_{\supset}$-бормуль $A$ верно следующее: $A$ есть $L_{\supset}$-формула, общезначимая в $L_{\supset}$-матрище $K$, тогда и только тогда, когда $A \in L$.

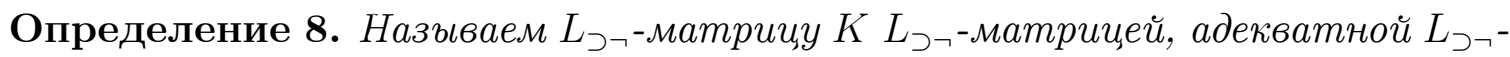

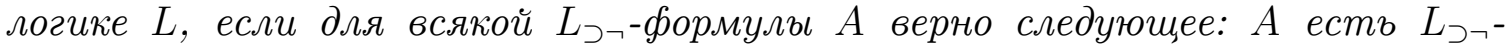

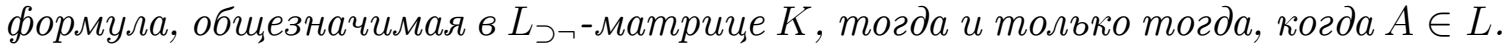

Соглашение 1. Обозначаем через $\supset_{(1,0,0,1 / 2)}$ бинарную операчию на $\{1,1 / 2,0\}$, определяемую следующей таблищей

\begin{tabular}{c|ccc}
$\supset(1,0,0,1 / 2)$ & 1 & $1 / 2$ & 0 \\
\hline 1 & 1 & 1 & 0 \\
$1 / 2$ & 1 & 1 & 0 \\
0 & 1 & $1 / 2$ & 1
\end{tabular}

Соглашение 2. Обозначаем через $\supset_{(1 / 2,1,0,1 / 2)}$ бинарную операчию на $\{1,1 / 2,0\}$, определяемую следующей таблищей

\begin{tabular}{c|ccc}
$\supset_{(1 / 2,1,0,1 / 2)}$ & 1 & $1 / 2$ & 0 \\
\hline 1 & 1 & $1 / 2$ & 1 \\
$1 / 2$ & 1 & 1 & 0 \\
0 & 1 & $1 / 2$ & 1
\end{tabular}

Соглашение 3. Обозначаем через $\supset_{C l}$ бинарную операцию на $\{0,1\}$, определяемую следующей таблищей

\begin{tabular}{c|cc}
$\supset_{C l}$ & 1 & 0 \\
\hline 1 & 1 & 0 \\
0 & 1 & 1
\end{tabular}

Соглашение 4. Обозначаем через $\neg$ Сl унарную операчию на $\{0,1\}$, определяемую следующей таблицей

$$
\begin{array}{l|ll}
\neg C l & 1 & 0 \\
\hline & 0 & 1
\end{array}
$$

Соглашение 5. Обозначаем через $s$ отображение множества $\{1,1 / 2,0\}$ на себл, определяемое следующей таблищей

$$
\begin{array}{c|ccc}
s & 1 & 1 / 2 & 0 \\
\hline & 1 & 0 & 1 / 2
\end{array}
$$


Соглашение 6. Обозначаем через $M_{(1,0,0,1 / 2)}$ упорядоченную тройку

$$
\langle\{1,1 / 2,0\},\{1\}, \supset(1,0,0,1 / 2)\rangle .
$$

Соглашение 7. Обозначаем через $M_{(1 / 2,1,0,1 / 2)}$ упорядоченную тройку

$$
\left\langle\{1,1 / 2,0\},\{1\}, \supset_{(1 / 2,1,0,1 / 2)}\right\rangle \text {. }
$$

Соглашение 8. Обозначаем через $M\left(C l_{\supset}\right)$ упорядоченную тройку $\langle\{0,1\},\{1\}$, $\left.\supset_{C l}\right\rangle$.

Соглашение 9. Обозначаем через $M\left(C l_{\supset \neg}\right)$ упорядоченную четверку

$$
\left\langle\{0,1\},\{1\}, \supset_{C l}, \neg_{C l}\right\rangle .
$$

Замечание 3. Упорядоченные тройки $M(1,0,0,1 / 2), M(1 / 2,1,0,1 / 2)$ u $M\left(C l_{\supset}\right)$

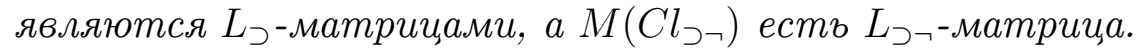

Соглашение 10. Обозначаем через $C l_{\supset}$ множество всех $L_{\supset}$-формул, общезначимых в $L_{\supset}$-матрице $M\left(C l_{\supset}\right)$.

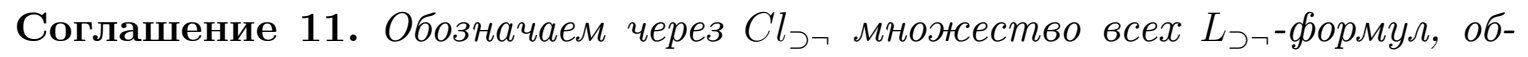

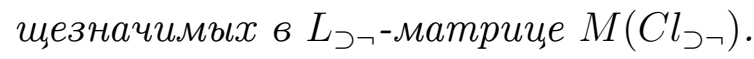

Замечание 4. Множество $C l_{\supset}$ всех $L_{\supset}$-формул, общезначимых в $L_{\supset}$-матрище $M\left(C l_{\supset}\right)$, есть $L_{\supset}$-логика.

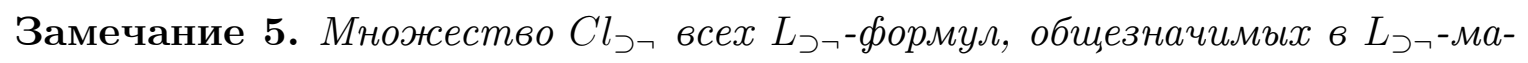

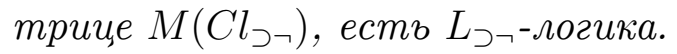

Замечание 6. Следуя традиции, называем $C l_{\supset}$ классической импликативной логикой в языке $L_{\supset}, a C l_{\supset\urcorner}-$ - лассической импликативно-негативной логикой в языке $L_{\supset \neg \text {. }}$.

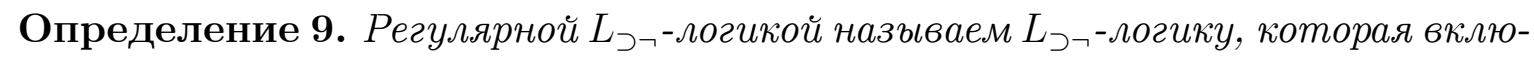
чается в $\mathrm{Cl}_{\supset \neg}$.

Определение 10. Называем s-преобразованием упорядоченной пары, первый член $q$ которой есть пропозициональная переменная языка $L_{\supset}$ и второй член $x$ которой есть элемент множества $\{1,1 / 2,0\}$, упорядоченную пару, первыи член которой есть q и второй член которой есть $s(x)$.

Определение 11. Называем s-преобразованием отображения $v$ множества всех пропозиииональных переменных языка $L_{\supset}$ в $1,1 / 2,0$ множество всех таких упорядоченных пар, каждая из которых есть s-преобразование упорядоченной парьи из $v$.

Замечание 7. Для всякой оценки языка $L_{\supset}$ в $L_{\supset}$-матрице $M(1,0,0,1 / 2)$ существует единственное $s$-преобразование этой оценки языка $L_{\supset}$ в $L_{\supset}$-матрице $M(1,0,0,1 / 2)$. 
Владимир Попов. К проблеме расширения матричной семантики...

Соглашение 12. Обозначаем s-преобразование ощенки v языка $L_{\supset}$ в $L_{\supset}$-матрииее $M(1,0,0,1 / 2)$ через $s[v]$.

Замечание 8. Для всякой оченки $v$ языка $L_{\supset}$ в $L_{\supset}$-матрище $M(1,0,0,1 / 2) s[v]$ является оценкой языка $L_{\supset}$ в $L_{\supset}$-матрице $M(1,0,0,1 / 2)$.

Лемма 1. Для всякой $L_{\supset}$ формуль $A: A$ есть пропозичиональная переменная языка $L_{\supset}$, или существует такое челое положительное число $n$, существуют такие $L_{\supset}$-формуль $A_{1}, \ldots, A_{n}$ и существует такая пропозициональная переменная $q$ языка $L_{\supset}$, что А есть $\left(A_{1} \supset\left(\ldots \supset\left(A_{n} \supset q\right) \ldots\right)\right)$.

Лемма 1 доказана индукцией по построению $L_{\supset}$-формулы.

Лемма 2. Для всякого иелого положительного числа $n$, для всяких $L_{\supset}$-формул $A_{1}, \ldots, A_{n}, B$ и для всякой оценки $v$ языка $L_{\supset}$ в $L_{\supset}$-матрице $M(1,0,0,1 / 2):$ если

$$
\varphi_{M}(1,0,0,1 / 2)\left(\left\langle\left(A_{1} \supset\left(\cdots \supset\left(A_{n} \supset B\right) \ldots\right)\right), v\right\rangle\right)=1 / 2,
$$

$$
\begin{aligned}
& \operatorname{mo} \varphi_{M}(1,0,0,1 / 2)\left(\left\langle A_{1}, v\right\rangle\right)=\ldots= \\
& \quad=\varphi_{M}(1,0,0,1 / 2)\left(\left\langle A_{n}, v\right\rangle\right)=0 u \varphi_{M}(1,0,0,1 / 2)(\langle B, v\rangle)=1 / 2 .
\end{aligned}
$$

Доказательство леммы 2 проводим прямой математической индукцией.

Базис. Для всякой $L_{\supset}$-формулы $A_{1}$, для всякой $L_{\supset}$-формулы $B$ и для всякой

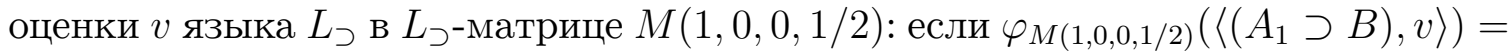
$1 / 2$, то $\varphi_{M(1,0,0,1 / 2)}\left(\left\langle\left(A_{1}\right), v\right\rangle\right)=0$ и $\varphi_{M(1,0,0,1 / 2)}(\langle(B), v\rangle)=1 / 2$. Справедливость базиса очевидна.

Индукционный шаг. Для всякого целого положительного числа $n$ : если для всяких $L_{\supset}$-формул $A_{1}, \ldots, A_{n}, B$ и для всякой оценки $v$ языка $L_{\supset}$ в $L_{\supset}$-матрице $M(1,0,0,1 / 2)$

$$
\begin{aligned}
\text { если } \varphi_{M}(1,0,0,1 / 2)\left(\left\langle\left(A_{1} \supset\left(\cdots \supset\left(A_{n} \supset B\right) \ldots\right)\right), v\right\rangle\right)=1 / 2, \\
\quad \text { то } \varphi_{M}(1,0,0,1 / 2)\left(\left\langle A_{1}, v\right\rangle\right)=\cdots=\varphi_{M}(1,0,0,1 / 2)\left(\left\langle A_{n}, v\right\rangle\right)=0 \\
\text { и } \varphi_{M}(1,0,0,1 / 2)(\langle B, v\rangle)=1 / 2,
\end{aligned}
$$

то для всяких $L_{\supset}$-формул $A_{1}, \ldots, A_{n}, A_{n+1}, B$ и для всякой оценки $v$ языка $L_{\supset}$

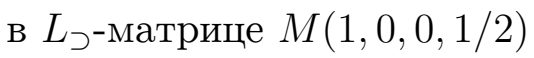

$$
\begin{aligned}
& \text { если } \varphi_{M}(1,0,0,1 / 2)\left(\left\langle\left(A_{1} \supset\left(\cdots \supset\left(A_{n} \supset\left(A_{n+1} \supset B\right)\right) \ldots\right)\right), v\right\rangle\right)=1 / 2, \\
& \quad \text { то } \varphi_{M}(1,0,0,1 / 2)\left(\left\langle A_{1}, v\right\rangle\right)=\cdots= \\
& \quad=\varphi_{M}(1,0,0,1 / 2)\left(\left\langle A_{n}, v\right\rangle\right)=\varphi_{M}(1,0,0,1 / 2)\left(\left\langle A_{n+1}, v\right\rangle\right)=0 \\
& \text { и } \varphi_{M}(1,0,0,1 / 2)(\langle B, v\rangle)=1 / 2 .
\end{aligned}
$$

Докажем индукционный шаг.

(1) $n^{\prime}$ есть целое положительное число (допущение). 
(2) Для всяких $L_{\supset}$-формул $A_{1}, \ldots, A_{n}, B$ и для всякой оценки $v$ языка $L_{\supset}$ в $L_{\supset}$-матрице $M(1,0,0,1 / 2)$

$$
\begin{aligned}
\text { если } \varphi_{M(1,0,0,1 / 2)}\left(\left\langle\left(A_{1} \supset\left(\cdots \supset\left(A_{n} \supset B\right) \ldots\right)\right), v\right\rangle\right)=1 / 2, \\
\quad \text { то } \varphi_{M(1,0,0,1 / 2)}\left(\left\langle A_{1}, v\right\rangle\right)=\cdots=\varphi_{M(1,0,0,1 / 2)}\left(\left\langle A_{n}, v\right\rangle\right)=0 \\
\text { и } \varphi_{M(1,0,0,1 / 2)}(\langle B, v\rangle)=1 / 2 \text { (допущение). }
\end{aligned}
$$

(3) $A_{1}^{\prime}, \ldots, A_{n^{\prime}}^{\prime}, A_{n^{\prime}+1}^{\prime}, B^{\prime}$ являются $L_{\supset}$-формулами (допущение).

(4) $v^{\prime}$ есть оценка языка $L_{\supset}$ в $L_{\supset}$-матрице $M(1,0,0,1 / 2)$ (допущение).

(5) $\varphi_{M(1,0,0,1 / 2)}\left(\left\langle\left(A_{1}^{\prime} \supset\left(\cdots \supset\left(A_{n^{\prime}}^{\prime} \supset\left(A_{n^{\prime}+1}^{\prime} \supset B^{\prime}\right)\right) \ldots\right)\right), v^{\prime}\right\rangle\right)=1 / 2$ (допущение).

(6) $A_{1}^{\prime}, \ldots, A_{n^{\prime}}^{\prime},\left(A_{n^{\prime}+1}^{\prime} \supset B^{\prime}\right)$ являются $L_{\supset}$-формулами (из $(3)$, по определению $L_{\supset}$-формулы).

(7) Если $\varphi_{M(1,0,0,1 / 2)}\left(\left\langle\left(A_{1}^{\prime} \supset\left(\ldots \supset\left(A_{n^{\prime}}^{\prime} \supset\left(A_{n^{\prime}+1}^{\prime} \supset B^{\prime}\right)\right) \ldots\right)\right), v^{\prime}\right\rangle\right)=1 / 2$, то

$$
\begin{aligned}
\varphi_{M(1,0,0,1 / 2)}\left(\left\langle A_{1}^{\prime}, v^{\prime}\right\rangle\right) & =\ldots=\varphi_{M(1,0,0,1 / 2)}\left(\left\langle A_{n^{\prime}}^{\prime}, v^{\prime}\right\rangle\right)=0 \\
\text { и } \varphi_{M(1,0,0,1 / 2)}\left(\left\langle\left(A_{n^{\prime}+1}^{\prime} \supset B^{\prime}\right), v^{\prime}\right\rangle\right) & =1 / 2(\text { из }(2),(4), \text { и }(6)) .
\end{aligned}
$$

Опираясь на базис и на утверждения (3) и (4), получаем, что

(8) если $\varphi_{M(1,0,0,1 / 2)}\left(\left\langle\left(A_{n^{\prime}+1}^{\prime} \supset B^{\prime}\right), v^{\prime}\right\rangle\right)=1 / 2$, то $\varphi_{M(1,0,0,1 / 2)}\left(\left\langle A_{n^{\prime}+1}^{\prime}, v^{\prime}\right\rangle\right)=0$ и

$$
\varphi_{M(1,0,0,1 / 2)}\left(\left\langle B^{\prime}, v^{\prime}\right\rangle\right)=1 / 2 \text {. }
$$

(9) Если $\varphi_{M(1,0,0,1 / 2)}\left(\left\langle\left(A_{1}^{\prime} \supset\left(\ldots \supset\left(A_{n^{\prime}}^{\prime} \supset\left(A_{n^{\prime}+1}^{\prime} \supset B^{\prime}\right)\right) \ldots\right)\right), v^{\prime}\right\rangle\right)=1 / 2$, то

$$
\begin{aligned}
& \varphi_{M(1,0,0,1 / 2)}\left(\left\langle A_{1}^{\prime}, v^{\prime}\right\rangle\right)=\cdots=\varphi_{M(1,0,0,1 / 2)}\left(\left\langle A_{n^{\prime}}^{\prime}, v^{\prime}\right\rangle\right)=\varphi_{M(1,0,0,1 / 2)}\left(\left\langle A_{n+1}, v^{\prime}\right\rangle\right)=0 \\
& \text { и } \left.\varphi_{M(1,0,0,1 / 2)}\left(\left\langle B^{\prime}, v^{\prime}\right\rangle\right)=1 / 2 \text { (из }(7) \text { и }(8)\right) .
\end{aligned}
$$

Снимая допущения (5), (4), (3), (2), (1) и проводя обобщения, завершаем доказательство индукционного шага.

Индукционный шаг доказан.

Лемма 2 доказана.

Лемма 3. Для всякой $L_{\supset}$ формуль $A$ и для всякой оценки $v$ языка $L_{\supset}$ в $L_{\supset^{-}}$ матрице $M(1,0,0,1 / 2)$ : если $\varphi_{M(1,0,0,1 / 2)}(\langle A, v\rangle)=0, \operatorname{mo} \varphi_{M(1,0,0,1 / 2)}(\langle A, s[v]\rangle) \in$ $\{1,1 / 2\}$.

Доказательство леммы 3 проводим индукцией по построению $L_{\supset}$-формулы.

Базис. Для всякой пропозициональной переменной $q$ языка $L_{\supset}$ и для всякой оценки $v$ языка $L_{\supset}$ в $L_{\supset}$-матрице $M(1,0,0,1 / 2)$ верно, что если $\varphi_{M(1,0,0,1 / 2)}(\langle q, v\rangle)=$ 0, то $\varphi_{M(1,0,0,1 / 2)}(\langle q, s[v]\rangle) \in\{1,1 / 2\}$.

Справедливость базиса очевидна. 
Владимир Попов. К проблеме расширения матричной семантики...

Индукционный шаг. Для всяких $L_{\supset}$-формул $B$ и $C$ : если ( для всякой оцен-

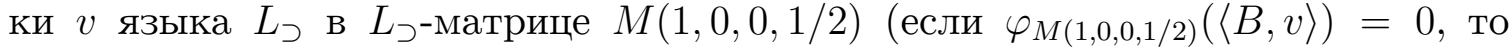

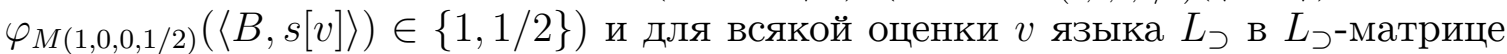
$M(1,0,0,1 / 2)$

$$
\text { если } \left.\varphi_{M(1,0,0,1 / 2)}(\langle C, v\rangle)=0, \text { то } \varphi_{M(1,0,0,1 / 2)}(\langle C, s[v]\rangle) \in\{1,1 / 2\}\right),
$$

то для всякой оценки $v$ языка $L_{\supset}$ в $L_{\supset}$-матрице $M(1,0,0,1 / 2)$ верно следующее: если $\varphi_{M(1,0,0,1 / 2)}(\langle(B \supset C), v\rangle)=0$, то $\varphi_{M(1,0,0,1 / 2)}(\langle(B \supset C), s[v]\rangle) \in\{1,1 / 2\}$.

Докажем индукционный шаг.

(1) $B_{0}$ есть $L_{\supset}$-формула (допущение).

(2) $C_{0}$ есть $L_{\supset}$-формула (допущение).

(3) Для всякой оценки $v$ языка $L_{\supset}$ в $L_{\supset}$-матрице $M(1,0,0,1 / 2)$

$$
\text { если } \varphi_{M(1,0,0,1 / 2)}\left(\left\langle B_{0}, v\right\rangle\right)=0, \text { то } \varphi_{M(1,0,0,1 / 2)}\left(\left\langle B_{0}, s[v]\right\rangle\right) \in\{1,1 / 2\}
$$

и для всякой оценки $v$ языка $L_{\supset}$ в $L_{\supset}$-матрице $M(1,0,0,1 / 2)$

если $\varphi_{M(1,0,0,1 / 2)}\left(\left\langle C_{0}, v\right\rangle\right)=0$, то $\varphi_{M(1,0,0,1 / 2)}\left(\left\langle C_{0}, s[v]\right\rangle\right) \in\{1,1 / 2\}$ (допущ.).

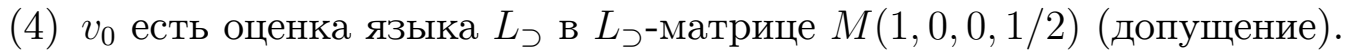

(5) $\varphi_{M(1,0,0,1 / 2)}\left(\left\langle\left(B_{0} \supset C_{0}\right), v_{0}\right\rangle\right)=0$ (допущение).

(6) $s\left[v_{0}\right]$ есть оценка языка $L_{\supset}$ в $L_{\supset}$-матрице $M(1,0,0,1 / 2)$ (из (4), по соглашению 12).

(7) Для всякой оценки $v$ языка $L_{\supset}$ в $L_{\supset}$-матрице $M(1,0,0,1 / 2)$ если $\varphi_{M(1,0,0,1 / 2)}\left(\left\langle B_{0}, v\right\rangle\right)=0$, то $\varphi_{M(1,0,0,1 / 2)}\left(\left\langle B_{0}, s[v]\right\rangle\right) \in\{1,1 / 2\}$ (из (3)).

(8) Для всякой оценки $v$ языка $L_{\supset}$ в $L_{\supset}$-матрице $M(1,0,0,1 / 2)$ если $\varphi_{M(1,0,0,1 / 2)}\left(\left\langle C_{0}, v\right\rangle\right)=0$, то $\varphi_{M(1,0,0,1 / 2)}\left(\left\langle C_{0}, s[v]\right\rangle\right) \in\{1,1 / 2\}$ (из (3)).

Опираясь на утверждения (1), (2), (4), (5), на замечание 1 и на соглашения 1 и 6 , получаем, что

(9) $\left(\varphi_{M(1,0,0,1 / 2)}\left(\left\langle B_{0}, v_{0}\right\rangle\right)=1\right.$ и $\left.\varphi_{M(1,0,0,1 / 2)}\left(\left\langle C_{0}, v_{0}\right\rangle\right)=0\right)$ и

$$
\left(\varphi_{M(1,0,0,1 / 2)}\left(\left\langle B_{0}, v_{0}\right\rangle\right)=1 / 2 \text { и } \varphi_{M(1,0,0,1 / 2)}\left(\left\langle C_{0}, v_{0}\right\rangle\right)=0\right) .
$$

(10) $\varphi_{M(1,0,0,1 / 2)}\left(\left\langle B_{0}, v_{0}\right\rangle\right)=1$ и $\varphi_{M(1,0,0,1 / 2)}\left(\left\langle C_{0}, v_{0}\right\rangle\right)=0$ (допущение).

(11) $\varphi_{M(1,0,0,1 / 2)}\left(\left\langle C_{0}, v_{0}\right\rangle\right)=0$ (из $\left.(10)\right)$. 
Ясно, что верно следующее утверждение (12).

(12) $\varphi_{M(1,0,0,1 / 2)}\left(\left\langle B_{0}, s\left[v_{0}\right]\right\rangle\right) \in\{1,1 / 2,0\}$.

(13) $\varphi_{M(1,0,0,1 / 2)}\left(\left\langle C_{0}, s\left[v_{0}\right]\right\rangle\right) \in\{1,1 / 2\}$ (из (8) и (11)).

Опираясь на утверждения (6), (12), (13), на замечание 1 и на соглашения 1 и 6 , получаем, что

(14) $\varphi_{M(1,0,0,1 / 2)}\left(\left\langle\left(B_{0} \supset C_{0}\right), s\left[v_{0}\right]\right\rangle\right) \in\{1,1 / 2\}$.

Снимая допущение (10), получаем, что

$(15)$ если $\varphi_{M(1,0,0,1 / 2)}\left(\left\langle B 0, v_{0}\right\rangle\right)=1$ и $\varphi_{M(1,0,0,1 / 2)}\left(\left\langle C_{0}, v_{0}\right\rangle\right)=0$, то

$$
\varphi_{M(1,0,0,1 / 2)}\left(\left\langle\left(B_{0} \supset C_{0}\right), s\left[v_{0}\right]\right\rangle\right) \in\{1,1 / 2\} .
$$

(16) $\varphi_{M(1,0,0,1 / 2)}\left(\left\langle B_{0}, v_{0}\right\rangle\right)=1 / 2$ и $\varphi_{M(1,0,0,1 / 2)}\left(\left\langle C_{0}, v_{0}\right\rangle\right)=0$ (допущение).

(17) $\varphi_{M(1,0,0,1 / 2)}\left(\left\langle C_{0}, v_{0}\right\rangle\right)=0$ (из $\left.(16)\right)$.

Ясно, что верно следующее утверждение (18).

(18) $\varphi_{M(1,0,0,1 / 2)}\left(\left\langle B_{0}, s\left[v_{0}\right]\right\rangle\right) \in\{1,1 / 2,0\}$.

(19) $\varphi_{M(1,0,0,1 / 2)}\left(\left\langle C_{0}, s\left[v_{0}\right]\right\rangle\right) \in\{1,1 / 2\}$ (из (8) и $\left.(18)\right)$.

Опираясь на утверждения (6), (18), (19), на замечание 1 и на соглашения 1 и 6 , получаем, что

(20) $\varphi_{M(1,0,0,1 / 2)}\left(\left\langle\left(B_{0} \supset C_{0}\right), s\left[v_{0}\right]\right\rangle\right) \in\{1,1 / 2\}$.

Снимая допущение (16), получаем, что

$(21)$ если $\varphi_{M(1,0,0,1 / 2)}\left(\left\langle B_{0}, v_{0}\right\rangle\right)=1 / 2$ и $\varphi_{M(1,0,0,1 / 2)}\left(\left\langle C_{0}, v_{0}\right\rangle\right)=0$, то

$$
\varphi_{M(1,0,0,1 / 2)}\left(\left\langle\left(B_{0} \supset C_{0}\right), s\left[v_{0}\right]\right\rangle\right) \in\{1,1 / 2\} .
$$

(22) $\varphi_{M(1,0,0,1 / 2)}\left(\left\langle\left(B_{0} \supset C_{0}\right), s\left[v_{0}\right]\right\rangle\right) \in\{1,1 / 2\}$ (из (9),(15) и $\left.(21)\right)$.

Снимая допущения (5), (4), (3), (2), (1) и проводя обобщения, завершаем доказательство индукционного шага.

Индукционный шаг доказан.

Лемма 3 доказана.

Лемма 4. Для всякого целого положителъного числа $n$, для всяких $L_{\supset}$-формул

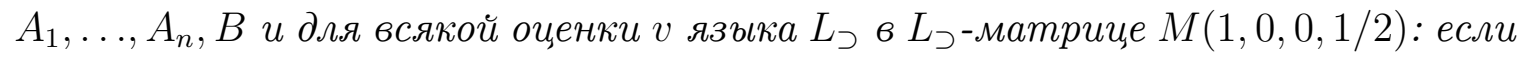

$$
\varphi_{M(1,0,0,1 / 2)}\left(\left\langle A_{1}, v\right\rangle\right), \ldots, \varphi_{M(1,0,0,1 / 2)}\left(\left\langle A_{n}, v\right\rangle\right)
$$

принадлежат множеству $\{1,1 / 2\}$ и $\varphi_{M(1,0,0,1 / 2)}(\langle B, v\rangle)=0$, то

$$
\varphi_{M(1,0,0,1 / 2)}\left(\left\langle\left(A_{1} \supset\left(\ldots \supset\left(A_{n} \supset B\right) \ldots\right)\right), v\right\rangle\right)=0 .
$$

Лемма 4 доказана прямой математической индукцией.

Лемма 5. Для всякой $L_{\supset}$ формулы $A$ и для всякой оценки $v$ языка $L_{\supset}$ в $L_{\supset}$-матрице $M(1,0,0,1 / 2)$ : если $\varphi_{M(1,0,0,1 / 2)}(\langle A, v\rangle)=1 / 2$, mо $\varphi_{M(1,0,0,1 / 2)}(\langle A, s[v]\rangle)=0$. 
Владимир Попов. К проблеме расширения матричной семантики...

Базис. Для всякой пропозициональной переменной $q$ языка $L_{\supset}$ и для вся-

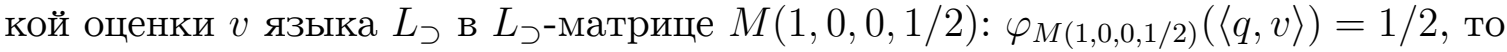
$\varphi_{M(1,0,0,1 / 2)}(\langle q, s[v]\rangle)=0$.

Справедливость базиса очевидна.

Индукционный шаг. Для всяких $L_{\supset}$-формул $B$ и $C$ :

если для всякой оценки $v$ языка $L_{\supset}$ в $L_{\supset}$-матрице $M(1,0,0,1 / 2)$

если $\varphi_{M(1,0,0,1 / 2)}(\langle B, v\rangle)=1 / 2$, то $\varphi_{M(1,0,0,1 / 2)}(\langle B, s[v]\rangle)=0$

и для всякой оценки $v$ языка $L_{\supset}$ в $L_{\supset}$-матрице $M(1,0,0,1 / 2)$

если $\varphi_{M(1,0,0,1 / 2)}(\langle C, v\rangle)=1 / 2$, то $\left.\left.\varphi_{M(1,0,0,1 / 2)}(\langle C, s[v]\rangle)=0\right)\right)$,

то для всякой оценки $v$ языка $L_{\supset}$ в $L_{\supset}$-матрице $M(1,0,0,1 / 2)$ верно:

если $\varphi_{M(1,0,0,1 / 2)}(\langle(B \supset C), v\rangle)=1 / 2$, то $\varphi_{M(1,0,0,1 / 2)}(\langle(B \supset C), s[v]\rangle)=0$.

Докажем индукционный шаг.

(1) $B_{0}$ есть $L_{\supset}$-формула (допущение).

(2) $C_{0}$ есть $L_{\supset}$-формула (допущение).

(3) Для всякой оценки $v$ языка $L_{\supset}$ в $L_{\supset}$-матрице $M(1,0,0,1 / 2)$

$$
\text { если } \left.\varphi_{M(1,0,0,1 / 2)}\left(\left\langle B_{0}, v\right\rangle\right)=1 / 2, \text { то } \varphi_{M(1,0,0,1 / 2)}\left(\left\langle B_{0}, s[v]\right\rangle\right)=0\right)
$$

и для всякой оценки $v$ языка $L_{\supset}$ в $L_{\supset}$-матрице $M(1,0,0,1 / 2)$

если $\left.\varphi_{M}(1,0,0,1 / 2)\left(\left\langle C_{0}, v\right\rangle\right\rangle\right)=1 / 2$, то $\left.\varphi_{M(1,0,0,1 / 2)}\left(\left\langle C_{0}, s[v]\right\rangle\right)=0\right)$ (допущ.).

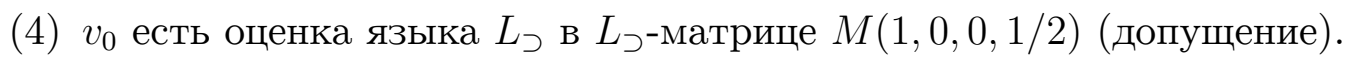

(5) $\varphi_{M(1,0,0,1 / 2)}\left(\left\langle\left(B_{0} \supset C_{0}\right), v_{0}\right\rangle\right)=1 / 2$ (допущение).

(6) $\varphi_{M(1,0,0,1 / 2)}\left(\left\langle B_{0}, v_{0}\right\rangle\right)=0$ и $\varphi_{M(1,0,0,1 / 2)}\left(\left\langle C_{0}, v_{0}\right\rangle\right)=1 / 2$ (из (1),(2) и (4), по лемме 2).

(7) $\varphi_{M(1,0,0,1 / 2)}\left(\left\langle B_{0}, v_{0}\right\rangle\right)=0$ (из $\left.(6)\right)$.

(8) $\varphi_{M(1,0,0,1 / 2)}\left(\left\langle C_{0}, v_{0}\right\rangle\right)=1 / 2$ (из $\left.(6)\right)$.

(9) $\varphi_{M(1,0,0,1 / 2)}\left(\left\langle B_{0}, s\left(v_{0}\right)\right\rangle\right) \in\{1,1 / 2\}$ (из $(1),(4)$ и $(7)$, по лемме 3$)$.

(10) $C_{0}$ есть пропозициональная переменная языка $L_{\supset}$, или существует такое целое положительное число $n$, существуют такие $L_{\supset}$-формулы $A_{1}, \ldots, A_{n}$ и существует такая пропозициональная переменная $q$ языка $L_{\supset}$, что $C_{0}$ есть $\left(A_{1} \supset\left(\ldots \supset\left(A_{n} \supset q\right) \ldots\right)\right)$ (из $(2)$, по лемме 1$)$.

(11) $C_{0}$ есть пропозициональная переменная языка $L_{\supset}$ (допущение). 
Опираясь на утверждения (4) и (11), а также на замечания 2 и 4, получаем, что

(12) $\varphi_{M(1,0,0,1 / 2)}\left(\left\langle C_{0}, v_{0}\right\rangle\right)=v_{0}\left(C_{0}\right)$.

(13) $v_{0}\left(C_{0}\right)=1 / 2($ из $(8)$ и $(12))$.

В свете утверждений (4), (13) и определения 3 ясно, что

(14) $\left\langle C_{0}, 1 / 2\right\rangle \in v_{0}$.

(15) $s\left[v_{0}\right]$ есть $s$-преобразование оценки $v_{0}$ языка $L_{\supset}$ в $L_{\supset}$-матрице $M(1,0,0,1 / 2)$ (из (4), по соглашению 8).

(16) $s\left[v_{0}\right]$ есть множество всех таких упорядоченных пар, каждая из которых есть $s$-преобразование упорядоченной пары из $v_{0}$ (из $(15)$, по определению 11).

Опираясь на утверждение (11), соглашение 5 и определение 10, получаем, что

(17) $\left\langle C_{0}, s(1 / 2)\right\rangle$ есть $s$-преобразование упорядоченной пары $\left\langle C_{0}, 1 / 2\right\rangle$.

Руководствуясь соглашением 5, получаем, что

(18) $s(1 / 2)=0$.

(19) $\left\langle C_{0}, 0\right\rangle$ есть $s$-преобразование упорядоченной пары $\left\langle C_{0}, 1 / 2\right\rangle$ (из (17) и (18)).

(20) $\left\langle C_{0}, 0\right\rangle \in s\left[v_{0}\right]$ (из (15), (16) и (19)).

(21) $s\left[v_{0}\right]$ есть оценка языка $L_{\supset}$ в $L_{\supset}$-матрице $M(1,0,0,1 / 2)$ (из $(15)$, по замечанию 8).

(22) $s\left[v_{0}\right]$ есть отображение множества всех пропозициональных переменных языка $L_{\supset}$ в $\{1,1 / 2,0\}$ (из (21), по определению 3 ).

(23) $s\left[v_{0}\right]\left(C_{0}\right)=0$ (из (11), (20) и $\left.(22)\right)$.

(24) $\varphi_{M(1,0,0,1 / 2)}\left(\left\langle C_{0}, s\left[v_{0}\right]\right\rangle\right)=s\left[v_{0}\right]\left(C_{0}\right)$ (из $(11)$ и $(21)$, по замечанию 1$)$.

(25) $\varphi_{M(1,0,0,1 / 2)}\left(\left\langle C_{0}, s\left[v_{0}\right]\right\rangle\right)=0$ (из $(23)$ и $\left.(24)\right)$.

Опираясь на утверждения (9), (25) и соглашение 1, получаем, что

(26) $\left.\varphi_{M(1,0,0,1 / 2)}\left(\left\langle B_{0}, s\left[v_{0}\right]\right\rangle\right) \supset_{(1,0,0,1 / 2)} \varphi_{M(1,0,0,1 / 2)}\left(\left\langle C_{0}, s\left[v_{0}\right]\right\rangle\right)\right)=0$.

(27) $\varphi_{M(1,0,0,1 / 2)}\left(\left\langle\left(B_{0} \supset C_{0}\right\rangle\right), s\left[v_{0}\right]\right)=$

$$
=\left(\varphi_{M(1,0,0,1 / 2)}\left(\left\langle B_{0}, s\left[v_{0}\right]\right\rangle\right) \supset_{(1,0,0,1 / 2)} \varphi_{M(1,0,0,1 / 2)}\left(\left\langle C_{0}, s\left[v_{0}\right]\right\rangle\right)\right)
$$

(из (1), (2), (21), по замечанию 1 и по замечанию 3 ).

(28) $\varphi_{M(1,0,0,1 / 2)}\left(\left\langle\left(B_{0} \supset C_{0}\right), s\left[v_{0}\right]\right\rangle\right)=0$ (из $(26)$ и $\left.(27)\right)$. 
Владимир Попов. К проблеме расширения матричной семантики...

Снимая допущение (11) получаем, что

(29) если $C_{0}$ есть пропозициональная переменная языка $L_{\supset}$, то

$$
\varphi_{M(1,0,0,1 / 2)}\left(\left\langle\left(B_{0} \supset_{(1,0,0,1 / 2)} C_{0}\right), s\left[v_{0}\right]\right\rangle\right)=0 .
$$

(30) Существует такое целое положительное число $n$, существуют такие $L_{\supset}$ формулы $A_{1}, \ldots, A_{n}$ и существует такая пропозициональная переменная $q$ языка $L_{\supset}$, что $C_{0}$ есть $\left(A_{1} \supset\left(\ldots \supset\left(A_{n} \supset q\right) \ldots\right)\right)$ (допущение).

Пусть

(31) $m$ есть целое положительное число, $A_{1}^{\prime}, \ldots, A_{m}^{\prime}$ являются $L_{\supset}$-формулами, $q^{\prime}$ есть пропозициональная переменная языка $L_{\supset}, C_{0}$ есть

$$
\left(A_{1}^{\prime} \supset\left(\cdots \supset\left(A_{m}^{\prime} \supset q^{\prime}\right) \ldots\right)\right) .
$$

(32) $m$ есть целое положительное число (из (31)).

(33) $A_{1}^{\prime}, \ldots, A_{m}^{\prime}$ являются $L_{\supset}$-формулами (из (31)).

(34) $q^{\prime}$ есть пропозициональная переменная языка $L_{\supset}$ (из (31)).

(35) $C_{0}$ есть $\left(A_{1}^{\prime} \supset\left(\ldots \supset\left(A_{m}^{\prime} \supset q^{\prime}\right) \ldots\right)\right)$ (из (31)).

(36) $\varphi_{M(1,0,0,1 / 2)}\left(\left(A_{1}^{\prime} \supset\left(\ldots \supset\left(A_{m}^{\prime} \supset q^{\prime}\right) \ldots\right)\right), s\left[v_{0}\right]\right)=1 / 2$ (из (8) и $\left.(35)\right)$.

(37) $q^{\prime}$ есть $L_{\supset}$-формула (из $(34)$, по определению $L_{\supset}$-формулы).

(38) $\varphi_{M(1,0,0,1 / 2)}\left(\left\langle A_{1}^{\prime}, s\left[v_{0}\right]\right\rangle\right)=\cdots=\varphi_{M(1,0,0,1 / 2)}\left(\left\langle A_{m}^{\prime}, s\left[v_{0}\right]\right\rangle\right)=0$ и

$$
\varphi_{M(1,0,0,1 / 2)}\left(\left\langle q^{\prime}, s\left[v_{0}\right]\right\rangle\right)=1 / 2 \text { (из (4), (32), (33), (37), по лемме 2). }
$$

(39) $\varphi_{M(1,0,0,1 / 2)}\left(\left\langle A_{1}^{\prime}, s\left[v_{0}\right]\right\rangle\right)=\cdots=\varphi_{M(1,0,0,1 / 2)}\left(\left\langle A_{m}^{\prime}, s\left[v_{0}\right]\right\rangle\right)=0$ (из (38)).

(40) $\varphi_{M(1,0,0,1 / 2)}\left(\left\langle q^{\prime}, s\left[v_{0}\right]\right\rangle\right)=1 / 2$ (из $\left.(38)\right)$.

Опираясь на утверждения (4), (37), замечания 1 и 3, получаем, что

(41) $\varphi_{M(1,0,0,1 / 2)}\left(\left\langle q^{\prime}, s\left[v_{0}\right]\right\rangle\right)=v_{0}\left(q^{\prime}\right)$.

(42) $v_{0}\left(q^{\prime}\right)=1 / 2($ из (40) и $(41))$.

Руководствуясь утверждением (42) и определением 3, получаем, что (43) $\left\langle q^{\prime}, 1 / 2\right\rangle \in v_{0}$.

Опираясь на утверждения (4), (43) и определения 10 и 11, получаем, что (44) $\left\langle q^{\prime}, s(1 / 2)\right\rangle \in s\left[v_{0}\right]$. 
(45) $s(1 / 2)=0$ (по соглашению 5).

(46) $\left\langle q^{\prime}, 0\right\rangle \in s\left[v_{0}\right]$ (из (44) и (45)).

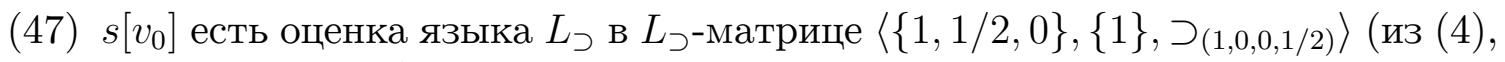
по замечанию 8).

(48) $s\left[v_{0}\right]$ есть отображение множества всех пропозициональных переменных языка $L_{\supset}$ в носитель $L_{\supset}$-матрицы $\left\langle\{1,1 / 2,0\},\{1\}, \supset_{(1,0,0,1 / 2)}\right\rangle$ (из $(47)$, по определению 3 ).

(49) $s\left[v_{0}\right]\left(q^{\prime}\right)=0$ (из (46) и (48)).

(50) $\varphi_{M(1,0,0,1 / 2)}\left(\left\langle q^{\prime}, s\left[v_{0}\right]\right\rangle\right)=0$ (из (34), (47), (49), по замечаниям 1 и 3).

(51) $\varphi_{M(1,0,0,1 / 2)}\left(\left\langle A_{1}^{\prime}, s\left[v_{0}\right]\right\rangle\right), \ldots, \varphi_{M(1,0,0,1 / 2)}\left(\left\langle A_{m}^{\prime}, s\left[v_{0}\right]\right\rangle\right) \in\{1,1 / 2\}$ (из (4), (33), (39), по лемме 3$)$.

(52) $\varphi_{M(1,0,0,1 / 2)}\left(\left\langle B_{0}, s\left[v_{0}\right]\right\rangle\right) \in\{1,1 / 2\}($ из $(1),(4),(7)$, по лемме 3$)$.

Опираясь на утверждения (1), (32), (33), (37), (47) и лемму 4, получаем, что

(53) $\varphi_{M(1,0,0,1 / 2)}\left(\left\langle\left(B_{0} \supset\left(A_{1}^{\prime} \supset\left(\cdots \supset\left(A_{m}^{\prime} \supset q^{\prime}\right) \cdots\right)\right)\right), s\left[v_{0}\right]\right\rangle\right)=0$.

(54) $\varphi_{M(1,0,0,1 / 2)}\left(\left\langle\left(B_{0} \supset C_{0}\right), s\left[v_{0}\right]\right\rangle\right)=0$ (из (35) и (53)).

Снимая допущение (30), получаем, что

(55) если существует такое целое положительное число $n$, существуют такие $L_{\supset}$ формулы $A_{1}, \ldots, A_{n}$ и существует такая пропозициональная переменная $q$ языка $L_{\supset}$, что $C_{0}$ есть $\left(A_{1} \supset\left(\ldots \supset\left(A_{n} \supset q\right) \ldots\right)\right)$, то

$$
\varphi_{M(1,0,0,1 / 2)}\left(\left\langle\left(B_{0} \supset C_{0}\right), s\left[v_{0}\right]\right\rangle\right)=0 .
$$

(56) $\varphi_{M(1,0,0,1 / 2)}\left(\left\langle\left(B_{0} \supset C_{0}\right), s\left[v_{0}\right]\right\rangle\right)=0$ (из $(10),(29)$ и $\left.(55)\right)$.

Снимая допущения (5), (4), (3), (2), (1) и проводя обобщения, завершаем доказательство индукционного шага.

Индукционный шаг доказан.

Лемма 5 доказана.

Лемма 6. Для всякой $L_{\supset}$ формулы $A$ и для всякой $L_{\supset}$ формуль В: если для

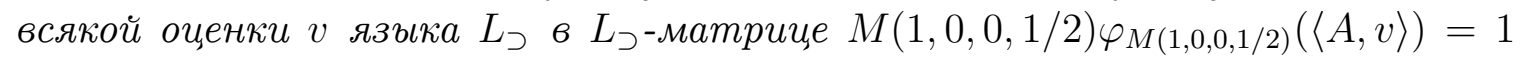

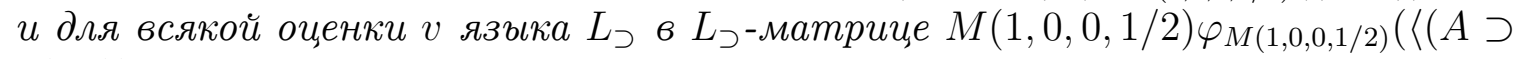

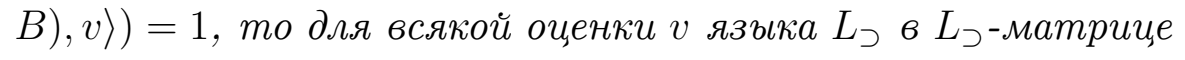

$$
M(1,0,0,1 / 2) \varphi_{M(1,0,0,1 / 2)}(\langle B, v\rangle)=1 .
$$

Докажем лемму 6. 
Владимир Попов. К проблеме расширения матричной семантики...

(1) $A_{0}$ есть $L_{\supset}$-формула (допущение).

(2) $B_{0}$ есть $L_{\supset}$-формула (допущение).

(3) Для всякой оценки $v$ языка $L_{\supset}$ в $L_{\supset}$-матрице

$$
M(1,0,0,1 / 2) \varphi_{M(1,0,0,1 / 2)}\left(\left\langle A_{0}, v\right\rangle\right)=1
$$

и для всякой оценки $v$ языка $L_{\supset}$ в $L_{\supset}$-матрице

$$
\left.M(1,0,0,1 / 2) \varphi_{M(1,0,0,1 / 2)}\left(\left\langle A_{0} \supset B_{0}\right), v\right\rangle\right)=1 \text { (допущение). }
$$

(4) Неверно, что для всякой оценки $v$ языка $L_{\supset}$ в $L_{\supset}$-матрице

$$
M(1,0,0,1 / 2) \varphi_{M(1,0,0,1 / 2)}\left(\left\langle B_{0}, v\right\rangle\right)=1 \text { (допущение). }
$$

(5) Существует такая оценка $v$ языка $L_{\supset}$ в $L_{\supset}$-матрице $M(1,0,0,1 / 2)$, что

$$
\left.\varphi_{M(1,0,0,1 / 2)}\left(\left\langle B_{0}, v\right\rangle\right) \neq 1 \text { (из }(4)\right) .
$$

Пусть

(6) $v_{0}$ есть оценка языка $L_{\supset}$ в $L_{\supset}$-матрице $M(1,0,0,1 / 2), \varphi_{M(1,0,0,1 / 2)}\left(\left\langle B_{0}, v_{0}\right\rangle\right) \neq$ 1.

Опираясь на утверждения (2) и (6) и на тот факт, что

$$
\varphi_{M(1,0,0,1 / 2)}\left(\left\langle B_{0}, v_{0}\right\rangle\right) \in\{1,1 / 2,0\},
$$

получаем, что

(7) $\varphi_{M(1,0,0,1 / 2)}\left(\left\langle B_{0}, v_{0}\right\rangle\right) \in\{1 / 2,0\}$.

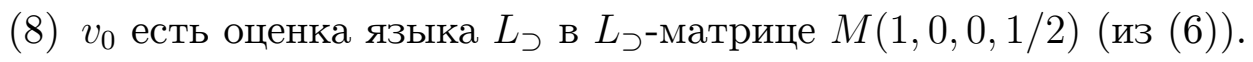

(9) $\varphi_{M(1,0,0,1 / 2)}\left(\left\langle B_{0}, v_{0}\right\rangle\right)=0$ или $\varphi_{M(1,0,0,1 / 2)}\left(\left\langle B_{0}, v_{0}\right\rangle\right)=1 / 2$ (из $\left.(7)\right)$.

(10) $\varphi_{M(1,0,0,1 / 2)}\left(\left\langle A_{0}, v_{0}\right\rangle\right)=1$ и $\varphi_{M(1,0,0,1 / 2)}\left(\left\langle\left(A_{0} \supset B_{0}\right), v_{0}\right\rangle\right)=1$ (из (3) и (8)).

(11) $\varphi_{M(1,0,0,1 / 2)}\left(\left\langle A_{0}, v_{0}\right\rangle\right)=1$ (из (10)).

(12) $\varphi_{M(1,0,0,1 / 2)}\left(\left\langle\left(A_{0} \supset B_{0}\right), v_{0}\right\rangle\right)=1$ (из (10)).

(13) $\varphi_{M(1,0,0,1 / 2)}\left(\left\langle B_{0}, v_{0}\right\rangle\right)=0$ (допущение).

В свете утверждений (8), (11), (13), замечания 1, соглашения 1 и того факта, что $M(1,0,0,1 / 2)$ есть $L_{\supset}$-матрица, ясно, что

(14) $\varphi_{M(1,0,0,1 / 2)}\left(\left\langle\left(A_{0} \supset B_{0}\right), v_{0}>\right)=0\right.$.

(15) $\varphi_{M(1,0,0,1 / 2)}\left(\left\langle\left(A_{0} \supset B_{0}\right), v_{0}\right\rangle\right) \neq 1$ (из $\left.(14)\right)$. 
Утверждение (15) противоречит утверждению (13). Следовательно, неверно допущение (13). Итак,

(16) $\varphi_{M(1,0,0,1 / 2)}\left(\left\langle B_{0}, v_{0}\right\rangle\right) \neq 0$.

(17) $\varphi_{M(1,0,0,1 / 2)}\left(\left\langle B_{0}, v_{0}\right\rangle\right)=1 / 2$ (из (9) и (16)).

(18) $\varphi_{M(1,0,0,1 / 2)}\left(\left\langle B_{0}, s\left[v_{0}\right]\right\rangle\right)=0$ (из (2), (8) и (17), по лемме 5).

(19) $s\left[v_{0}\right]$ есть оценка языка $L_{\supset}$ в $L_{\supset}$-матрице $M(1,0,0,1 / 2)$ (из $(8)$, по замечанию 8).

(20) $\varphi_{M(1,0,0,1 / 2)}\left(\left\langle A_{0}, s\left[v_{0}\right]\right\rangle\right)=1$ и $\left.\varphi_{M(1,0,0,1 / 2)}\left(\left\langle A_{0} \supset B_{0}\right), s\left[v_{0}\right]\right\rangle\right)=1$ (из (3) и $\left.(19)\right)$.

(21) $\varphi_{M(1,0,0,1 / 2)}\left(\left\langle A_{0}, s\left[v_{0}\right]\right\rangle\right)=1$ (из (20)).

(22) $\varphi_{M(1,0,0,1 / 2)}\left(\left\langle\left(A_{0} \supset B_{0}\right), s\left[v_{0}\right]\right\rangle\right)=1$ (из $\left.(20)\right)$.

В свете утверждений (18), (19), (21), замечаний 1 и 3 и соглашения 1 ясно, что

(23) $\varphi_{M(1,0,0,1 / 2)}\left(\left\langle\left(A_{0} \supset B_{0}\right), s\left[v_{0}\right]\right\rangle\right)=0$.

(24) $\varphi_{M(1,0,0,1 / 2)}\left(\left\langle\left(A_{0} \supset B_{0}\right), s\left[v_{0}\right]\right\rangle\right) \neq 1$ (из $\left.(23)\right)$.

Утверждение (24) противоречит утверждению (22). Следовательно, неверно допущение (4). Но тогда

(25) Для всякой оценки $v$ языка $L_{\supset}$ в $L_{\supset}$-матрице $M(1,0,0,1 / 2)$

$$
\varphi_{M(1,0,0,1 / 2)}\left(\left\langle B_{0}, v\right\rangle\right)=1 \text {. }
$$

Снимая допущения (3), (2), (1) и проводя обобщения, завершаем доказательство леммы 6.

Лемма 6 доказана.

Нам потребуется исчисление $H C l_{\supset}$ гильбертовского типа. Языком этого исчисления является $L_{\supset}$. Правило modus ponens в $L_{\supset}$ есть единственное правило исчисления $\mathrm{HCl}_{\supset}$. Аксиомами исчисления $\mathrm{HCl}_{\supset}$ являются все те и только те $L_{\supset}$-формулы, каждая из которых имеет хотя бы один из следующих трех видов (здесь $A, B$ и $C-L_{\supset}$-формулы): (1) $(A \supset(B \supset A)),(2)((A \supset(B \supset C)) \supset((A \supset$

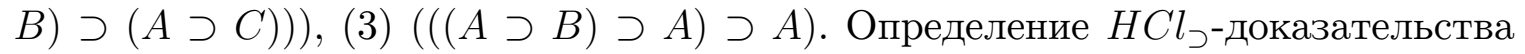
$L_{\supset}$-формулы: $\alpha$ есть $H C l_{\supset}$-доказательство $L_{\supset}$-формулы $A$, если существуют такое целое положительное число $n$ и такие $L_{\supset}$-формулы $A_{1}, \ldots, A_{n}$, что $\alpha$ есть $n$-членная последовательность $L_{\supset}$-формул, первый член которой есть $A_{1}, \ldots, n$ ный член которой есть $A_{n}$, и выполняются следующие два условия: (I) $A_{n}$ есть $A,(\mathrm{II})$ для всякого целого положительного числа $i$, которое $\leq n, A_{i}$ есть аксиома исчисления $H C l_{\supset}$ или существуют такие целые положительные числа $k$ и $l$, каждое из которых меньше $i$, что $\left\langle A_{k}, A_{l}, A_{n}\right\rangle$ есть применение правила modus ponens в $L_{\supset}$.

Опираясь на (Соболев 1979) и на (Черч 1960), можно доказать следующее утверждение. 
Владимир Попов. К проблеме расширения матричной семантики...

Утверждение 1. Для всякой $L_{\supset}$-ормулы $A: A \in C l_{\supset}$ тогда и толъко тогда, когда существует $\mathrm{HCl}_{\supset}$-доказательство $L_{\supset}$-формулы $A$.

Легко установить, что верна следующая лемма 7.

Лемма 7. Для всякой аксиомы $A$ исчисления $H C l_{\supset}$ и для всякой оценки $v$

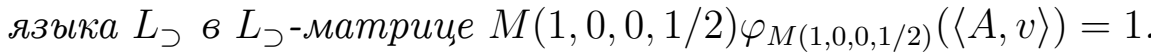

Лемма 8. Для всякого иелого положительного числа $n$ и для всяких $L_{\supset}$ формул $A_{1}, \ldots, A_{n}$ : если для всякого иелого положительного числа $i$, которое $\leq n, A_{i}$ есть аксиома исчисления $\mathrm{HCl}_{\supset}$ или существуют такие иелые положительные числа $k$ и $l$, каждое из которых меньше $i$, что $\left\langle A_{k}, A_{l}, A_{n}\right\rangle$ есть применение правила modus ponens в $L_{\supset}$, то для всякой оценки $v$ языка $L_{\supset} в$

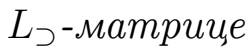

$$
M(1,0,0,1 / 2) \varphi_{M(1,0,0,1 / 2)}\left(\left\langle A_{n}, v\right\rangle\right)=1 .
$$

Стандартное индуктивное доказательство (методом возвратной индукции) леммы 8 проведено с использованием леммы 6 и леммы 7.

Лемма 9. Для всякой $L_{\supset}$-формуль $A$ : если существует $H C l_{\supset}$-доказательство $L_{\supset}$-ормулы $A$, то для всякой оценки $v$ языка $L_{\supset}$ в $L_{\supset}$-матрице

$$
M(1,0,0,1 / 2) \varphi_{M(1,0,0,1 / 2)}(\langle A, v\rangle)=1 .
$$

Докажем лемму 9.

(1) $A_{0}$ есть $L_{\supset}$-формула (допущение).

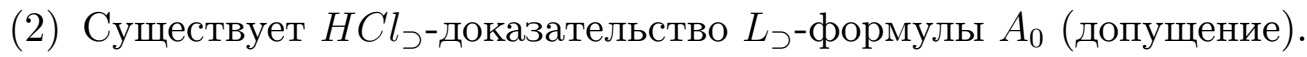

Пусть

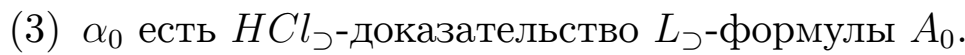

(4) Существуют такое целое положительное число $n$ и такие $L_{\supset}$-формулы $A_{1}, \ldots, A_{n}$, что $\alpha_{0}$ есть $n$-членная последовательность $L_{\supset}$-формул, первый член которой есть $A_{1}$, и ..., и $n$-ный член которой есть $A_{n}$, и выполняются следующие два условия: (I) $A_{n}$ есть $A_{0}$, (II) для всякого целого положительного числа $i$, которое $\leq n, A_{i}$ есть аксиома исчисления $H C l_{\supset}$ или существуют такие целые положительные числа $k$ и $l$, каждое из которых меньше $i$, что $\left\langle A_{k}, A_{l}, A_{n}\right\rangle$ есть применение правила modus ponens в $L_{\supset}$ (из (3), по определению $H C l_{\supset}$-доказательства $L_{\supset}$-формулы).

(5) Существуют такое целое положительное число $n$ и такие $L_{\supset}$-формулы $A_{1}, \ldots, A_{n}$, что для всякого целого положительного числа $i$, которое $\leq n$, $A_{i}$ есть аксиома исчисления $H C l_{\supset}$ или существуют такие целые положительные числа $k$ и $l$, каждое из которых меньше $i$, что $\left\langle A_{k}, A_{l}, A_{n}\right\rangle$ есть применение правила modus ponens в $L_{\supset}$ (из (4)). 
Пусть

(6) $m$ есть целое положительное число, $A_{1}^{\prime}, \ldots, A_{m}^{\prime}$ являются $L_{\supset}$-формулами, для всякого целого положительного числа $i$, которое $\leq n, A_{i}$ есть аксиома исчисления $H C l_{\supset}$ или существуют такие целые положительные числа $k$ и $l$, каждое из которых меньше $i$, что $\left\langle A_{k}, A_{l}, A_{n}\right\rangle$ есть применение правила modus ponens в $L_{\supset}$.

(7) Для всякой оценки $v$ языка $L_{\supset}$ в $L_{\supset}$-матрице $M(1,0,0,1 / 2)$

$$
\varphi_{M(1,0,0,1 / 2)}\left(\left\langle A_{m}^{\prime}, v\right\rangle\right)=1 \text { (из }(6), \text { по лемме 8). }
$$

Снимая допущения (2), (1) и обобщая, завершаем доказательство леммы 9. Лемма 9 доказана.

Лемма 10. Для всякой $L_{\supset}$ формуль $A$ : если $A \in C l_{\supset}$, то $A$ есть $L_{\supset}$-формула, общезначимая в $L_{\supset}$-матрище $M(1,0,0,1 / 2)$.

Докажем лемму 10.

(1) $A_{0}$ есть $L_{\supset}$-формула (допущение).

(2) $A_{0} \in C l_{\supset}$ (допущение).

(3) Существует $H C l_{\supset}$-доказательство $L_{\supset}$-формулы $A_{0}$ (из $(1),(2)$ и утверждения).

(4) Для всякой оценки $v$ языка $L_{\supset}$ в $L_{\supset}$-матрице $M(1,0,0,1 / 2)$

$$
\left.\varphi_{M(1,0,0,1 / 2)}\left(\left\langle A_{0}, v\right\rangle\right)=1 \text { (из }(1) \text { и }(3), \text { по лемме } 9\right) \text {. }
$$

Опираясь на определение 1 , на соглашение 6 и на замечание 3 , получаем, что

(5) множество $\{1\}$ есть выделенное множество $L_{\supset}$-матрицы $M(1,0,0,1 / 2)$.

В свете утверждений (4) и (5) ясно, что

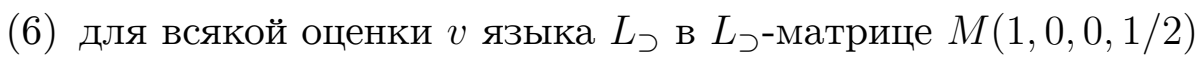

$$
\varphi_{M(1,0,0,1 / 2)}\left(\left\langle A_{0}, v\right\rangle\right)
$$

принадлежит выделенному множеству $L_{\supset}$-матрицы $M(1,0,0,1 / 2)$.

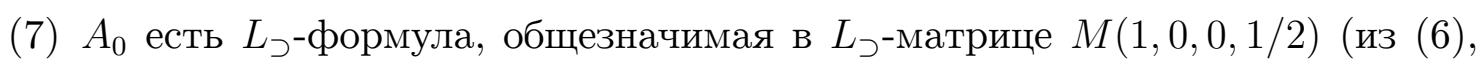
по определению 5).

Снимая допущения (2) и (1) и обобщая, завершаем доказательство леммы 10.

Лемма 10 доказана.

Легко установить справедливость следующей леммы 11. 
Владимир Попов. К проблеме расширения матричной семантики...

Лемма 11. Для всякой $L_{\supset}$ формулы $A$ и для всякой оценки $v$ языка $L_{\supset}$ в

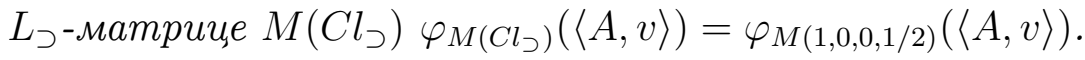

Лемма 12. Для всякой $L_{\supset}$ формулы $A$ : если для всякой оченки $v$ языка $L_{\supset} в$

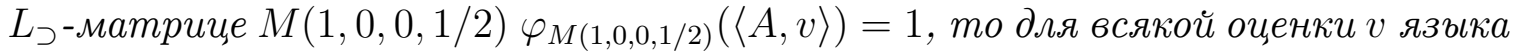

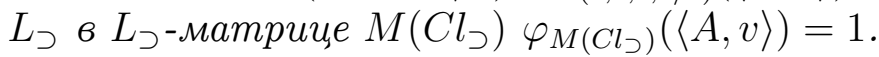

Докажем лемму 12.

(1) $A_{0}$ есть $L_{\supset}$-формула (допущение).

(2) Для всякой оценки $v$ языка $L_{\supset}$ в $L_{\supset}$-матрице $M(1,0,0,1 / 2)$

$$
\varphi_{M(1,0,0,1 / 2)}\left(\left\langle A_{0}, v\right\rangle\right)=1 \text { (допущение) }
$$

(3) Существует такая оценка $v$ языка $L_{\supset}$ в $L_{\supset}$-матрице $M\left(C l_{\supset}\right)$, что

$$
\varphi_{M\left(C l_{\supset}\right)}\left(\left\langle A_{0}, v\right\rangle\right) \neq 1 \text { (допущение). }
$$

Пусть

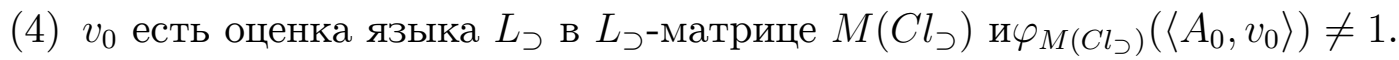

(5) $\varphi_{M(1,0,0,1 / 2)}\left(\left\langle A_{0}, v_{0}\right\rangle\right) \neq 1$ (из (1) и (4), по лемме 9).

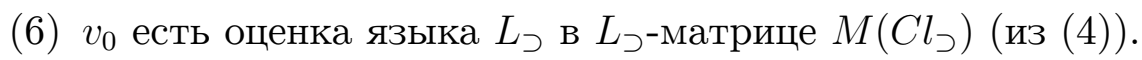

(7) $v_{0}$ есть оценка языка $L_{\supset}$ в $L_{\supset}$-матрице $M(1,0,0,1 / 2)$ (из (4) и из того, что всякая оценка языка $L_{\supset}$ в $L_{\supset}$-матрице $M\left(C l_{\supset}\right)$ является оценкой языка

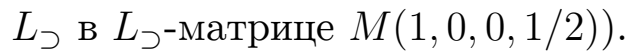

(8) $\varphi_{M(1,0,0,1 / 2)}\left(\left\langle A_{0}, v_{0}\right\rangle\right)=1$ (из $(2)$ и $\left.(7)\right)$.

Утверждение (8) противоречит утверждению (5). Следовательно, неверно допущение (3). Но тогда верно, что (9) для всякой оценки $v$ языка $L_{\supset}$ в $L_{\supset}$-матрице $M\left(C l_{\supset}\right) \varphi_{M\left(C l_{\supset}\right)}(\langle A, v\rangle)=1$.

Снимая допущения (2), (1) и обобщая, завершаем доказательство леммы 12.

Лемма 12 доказана.

Опираясь на определение 1 и 5, на соглашения 6,8 и 10, на замечание 3 и на лемму 12, приходим к выводу, что верна следующая лемма 13.

Лемма 13. Для всякой $L_{\supset}$ формуль $A$ : если $A$ есть $L_{\supset}$-формула, общезначимая в $L_{\supset}$-матриче $M(1,0,0,1 / 2)$, то $A \in C l_{\supset}$.

Из лемм 10 и 13 вытекает следующая теорема 1.

Теорема 1. Для всякой $L_{\supset}$ формулы $A: A \in C l_{\supset}$ тогда и толъко тогда, когда $A$ есть $L_{\supset}$-бормула, общезначимая в $L_{\supset}$-матрище $M(1,0,0,1 / 2)$. 
Наша ближайшая цель - доказательство того, что не существует унарной операции $f$ на множестве $\{1,1 / 2,0\}$, выполняющей условие: упорядоченная

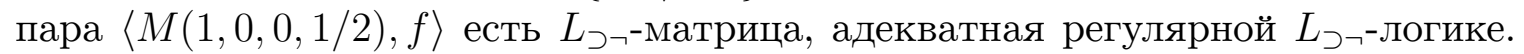
Для достижения указанной цели достаточно доказать, что для всякой унарной операции $f$ на множестве $\{1,1 / 2,0\}$ упорядоченная пара $\langle M(1,0,0,1 / 2), f\rangle$ есть

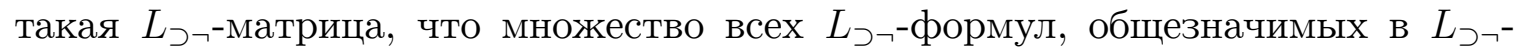

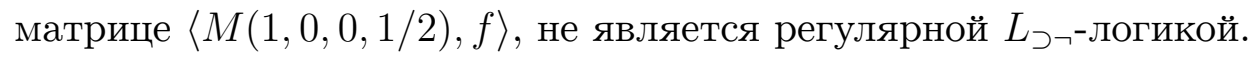

Замечание 9. Для всяких $x, y$ u z из $\{1,1 / 2,0\}$ существует единственная унарная операция $f$ на $\{1,1 / 2,0\}$, удовлетворяющая условиям: (1) $f(1)=x$, (2) $f(1 / 2)=y$, (3) $f(0)=z$.

Соглашение 13. Обозначаем через $\neg(x, y, z)$ mу самую унарную операчию $f$ на множестве $\{1,1 / 2,0\}$, которая удовлетворяет условиям: (1) $f(1)=x$, (2) $f(1 / 2)=y$, (3) $f(0)=z$.

Лемма 14. Для всякой унарной операции $f$ на множестве $\{1,1 / 2,0\}$ : упо-

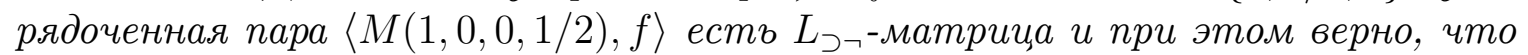

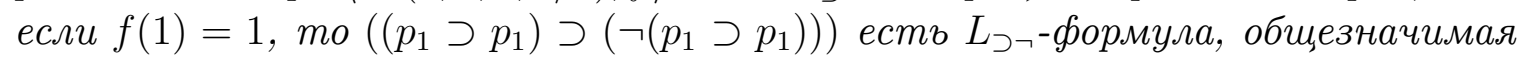

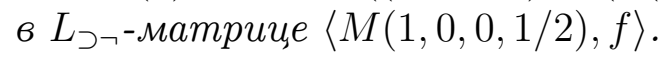

Лемма 15. Для всякой унарной операции $f$ на множестве $\{1,1 / 2,0\}$ : упо-

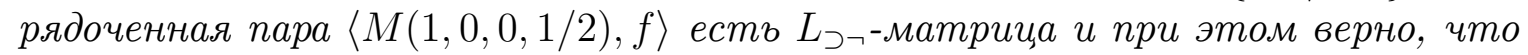

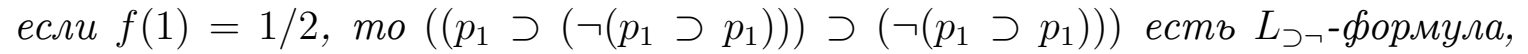

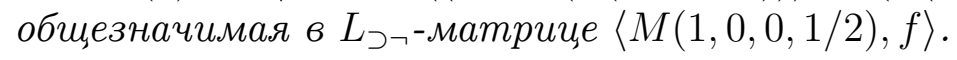

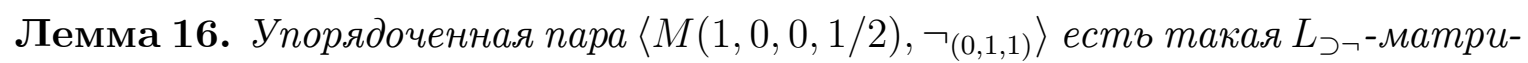
и,а, что $\left(\left(p_{1} \supset p_{1}\right) \supset\left(\left(\neg\left(\neg p_{1}\right)\right) \supset p_{1}\right)\right)$ u $\left(p_{1} \supset p_{1}\right)$ являются $L_{\supset \neg}$-формулами,

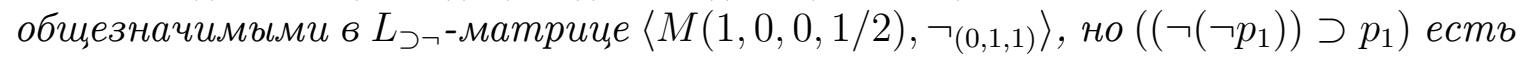

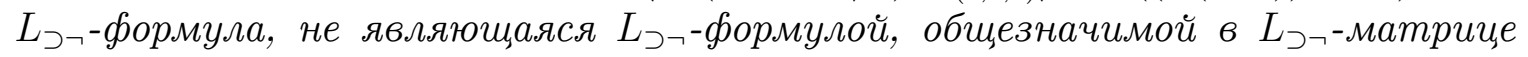
$\langle M(1,0,0,1 / 2), \neg(0,1,1)\rangle$.

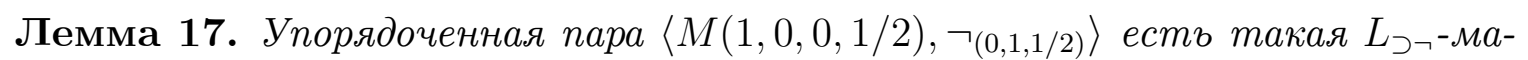

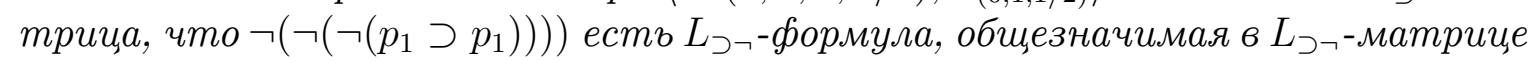

$$
\langle M(1,0,0,1 / 2), \neg(0,1,1 / 2)\rangle .
$$

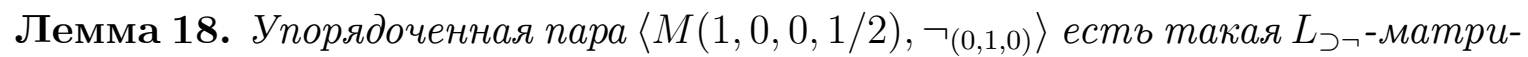

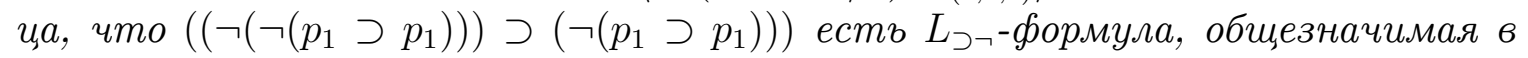
$\left.L_{\supset \neg \text {-мampuщ, }\langle M(1,0,0,1 / 2), \neg(0,1,0)}\right\rangle$.

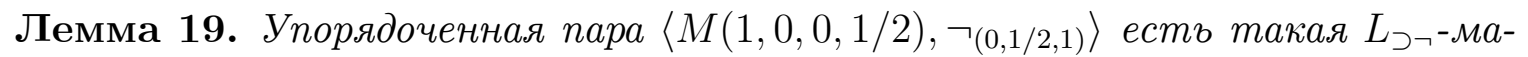
трица, что $\left(\left(p_{1} \supset p_{1}\right) \supset\left(\left(p_{1} \supset\left(\neg\left(p_{1} \supset p_{1}\right)\right)\right) \supset\left(\neg p_{1}\right)\right)\right)$ u $\left(p_{1} \supset p_{1}\right)$ являются

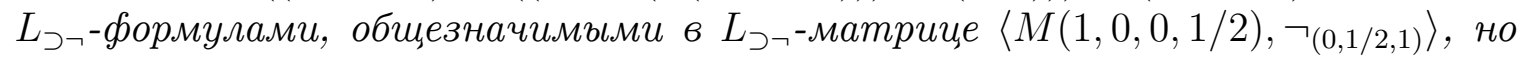
$\left(\left(p_{1} \supset\left(\neg\left(p_{1} \supset p_{1}\right)\right)\right) \supset\left(\neg p_{1}\right)\right)$ есть $L_{\supset \neg}$-бормула, не являющаяся $L_{\supset \neg-}$ бормулой,

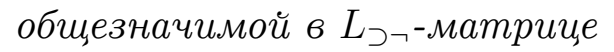

$$
\langle M(1,0,0,1 / 2), \neg(0,1 / 2,1)\rangle .
$$


Владимир Попов. К проблеме расширения матричной семантики...

Лемма 20. Упорядоченная пара $\langle M(1,0,0,1 / 2), \neg(0,1 / 2,1 / 2)\rangle$ есть такая $L_{\supset \neg}$ матриц, ат, что $\left(\left(p_{1} \supset\left(\neg\left(\neg\left(\neg\left(p_{1} \supset p_{1}\right)\right)\right)\right)\right) \supset\left(\neg\left(\neg\left(\neg\left(p_{1} \supset p_{1}\right)\right)\right)\right)\right)$ есть $L_{\supset \neg^{-}}$ бормула, общезначимая в $L_{\supset \neg-м а т р и щ е ~}\langle M(1,0,0,1 / 2), \neg(0,1 / 2,1 / 2)\rangle$.

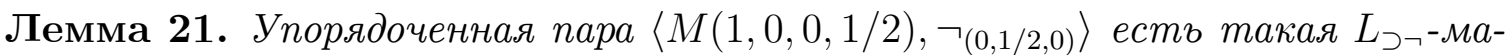
трича, что $\left(\left(\neg\left(\neg\left(p_{1} \supset p_{1}\right)\right)\right) \supset\left(\neg\left(p_{1} \supset p_{1}\right)\right)\right)$ есть $L_{\supset \neg-}$ бормула, общезначимая

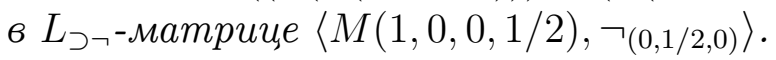

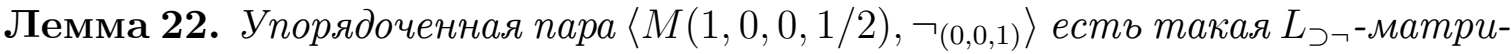

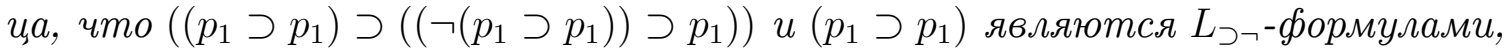

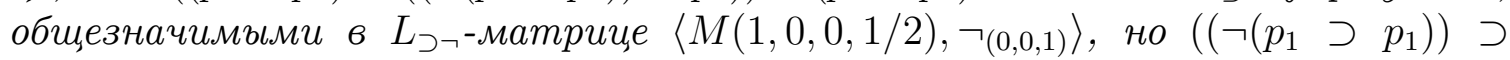
$\left.p_{1}\right)$ есть $L_{\supset\urcorner^{-}}$бормула, не являющаяся $L_{\supset \neg-}$-бормулой, общезначимой в $L_{\supset \neg^{-}}$ матрище $\langle M(1,0,0,1 / 2), \neg(0,0,1)\rangle$.

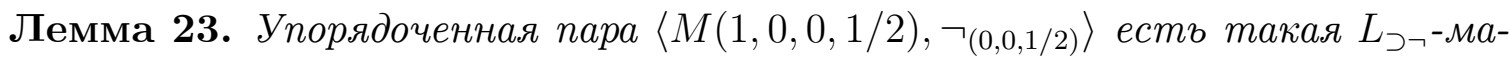

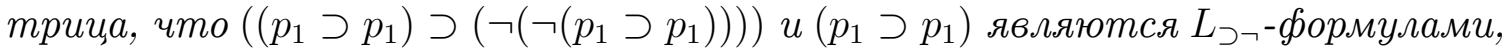

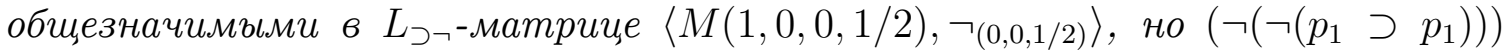

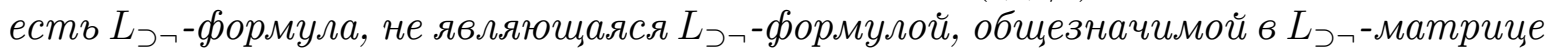
$\langle M(1,0,0,1 / 2), \neg(0,0,1 / 2)\rangle$.

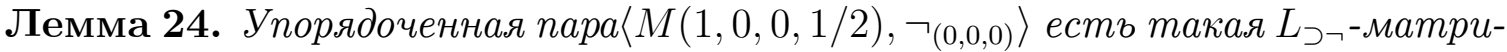

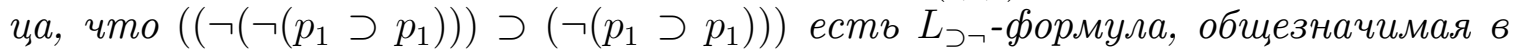
$\left.L_{\supset \neg-\text {-мampu-u,e }\langle M(1,0,0,1 / 2), \neg(0,0,0)}\right\rangle$.

Проверка того, что все леммы 14-24 верны, основанная на процедуре построения соответствующих истинностных таблиц, не представляет труда.

Теорема 2. Для всякой унарной операчии $f$ на множестве $\{1,1 / 2,0\}$ упоря-

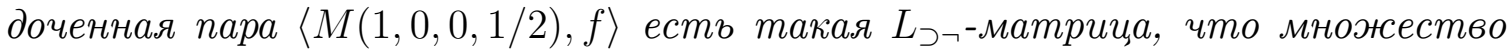
всех $L_{\supset \neg-}$ бормул, общезначимых в $L_{\supset \neg-м а т р и щ е ~}\langle M(1,0,0,1 / 2), f\rangle$, не явля-

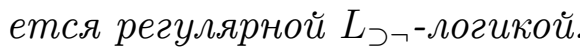

Докажем теорему 2.

(1) $f_{0}$ есть унарная операция на множестве $\{1,1 / 2,0\}$ (допущение).

Очевидно, что

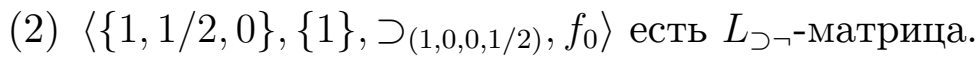

Опираясь на утверждение (2) и тот факт, что

$$
\left\langle\{1,1 / 2,0\},\{1\}, \supset_{(1,0,0,1 / 2)}, f_{0}\right\rangle=\left\langle\left\langle\{1,1 / 2,0\},\{1\}, \supset_{(1,0,0,1 / 2)}\right\rangle, f_{0}\right\rangle,
$$

получаем, что

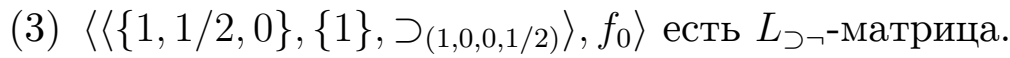

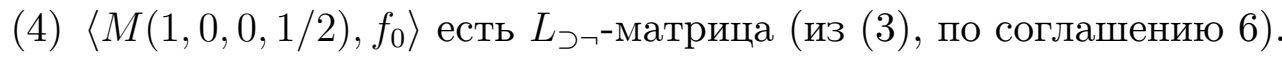


Принимая во внимание утверждение (1), приходим к выводу, что

(5) $f_{0}(1)=1$, или $f_{0}(1)=1 / 2$, или $f_{0}(1)=0$.

(6) $f_{0}(1)=1$ (допущение).

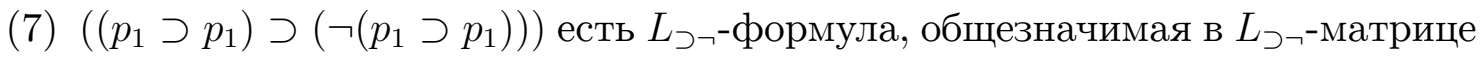

$$
\left\langle M(1,0,0,1 / 2), f_{0}\right\rangle
$$

(из (1) и (6), по лемме 14).

Легко убедиться, что

(8) $\left(\left(p_{1} \supset p_{1}\right) \supset\left(\neg\left(p_{1} \supset p_{1}\right)\right)\right)$ не есть $L_{\supset \neg}$-формула, общезначимая в $L_{\supset\urcorner^{-}}$ матрице $M\left(C l_{\supset\urcorner}\right)$.

Опираясь на утверждение (8) и соглашение 11 , получаем, что

(9) $\left(\left(p_{1} \supset p_{1}\right) \supset\left(\neg\left(p_{1} \supset p_{1}\right)\right)\right)$ не принадлежит множеству $C l_{\supset \neg \text {. }}$.

В свете утверждений (7) и (9) ясно, что

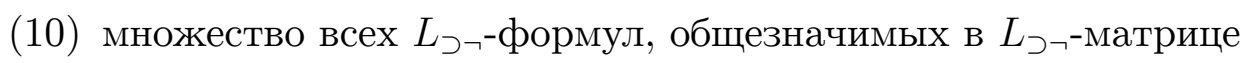

$$
\left\langle M(1,0,0,1 / 2), f_{0}\right\rangle
$$

не включается в $C l_{\supset \neg}$.

(11) Множество всех $L_{\supset\urcorner}$-формул, общезначимых в $L_{\supset\urcorner}$-матрице

$$
\left\langle M(1,0,0,1 / 2), f_{0}\right\rangle
$$

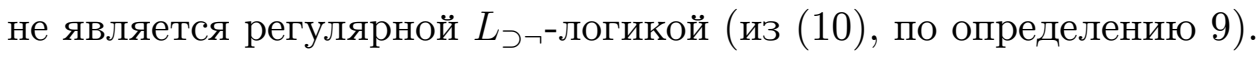

Снимая допущение (6), получаем, что

(12) если $f_{0}(1)=1$, то множество всех $L_{\supset\urcorner}$-формул, общезначимых в $L_{\supset\urcorner^{-}}$ матрице $\left\langle M(1,0,0,1 / 2), f_{0}\right\rangle$, не является регулярной $L_{\supset-}$-логикой.

(13) $f_{0}(1)=1 / 2$ (допущение).

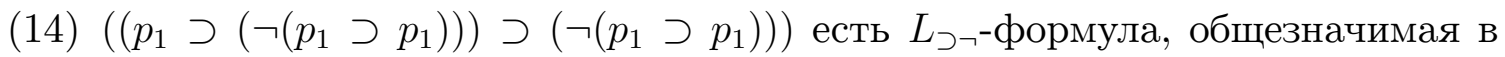

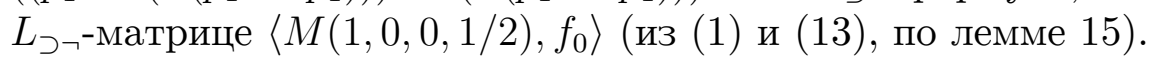

Легко убедиться, что

(15) $\left(\left(p_{1} \supset\left(\neg\left(p_{1} \supset p_{1}\right)\right)\right) \supset\left(\neg\left(p_{1} \supset p_{1}\right)\right)\right)$ не есть $L_{\supset-}$-формула, общезначимая в

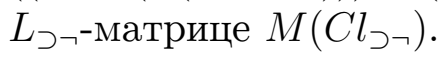

Опираясь на утверждение (15) и соглашение 11, получаем, что 
Владимир Попов. К проблеме расширения матричной семантики...

(16) $\left(\left(p_{1} \supset\left(\neg\left(p_{1} \supset p_{1}\right)\right)\right) \supset\left(\neg\left(p_{1} \supset p_{1}\right)\right)\right)$ не принадлежит множеству $C l_{\supset \neg}$

В свете утверждений (15) и (16) ясно, что

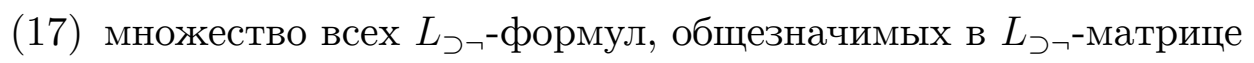

$$
\left\langle M(1,0,0,1 / 2), f_{0}\right\rangle
$$

не включается в $C l_{\supset \neg}$.

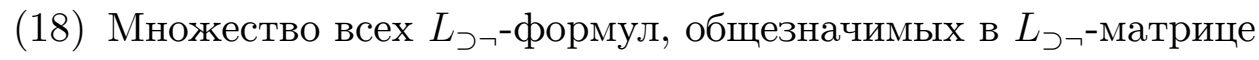

$$
\left\langle M(1,0,0,1 / 2), f_{0}\right\rangle
$$

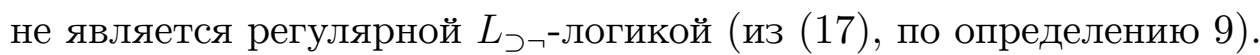

Снимая допущение (13), получаем, что

(19) если $f_{0}(1)=1 / 2$, то множество всех $L_{\supset-}$-формул, общезначимых в $L_{\supset\urcorner^{-}}$ матрице $\left\langle M(1,0,0,1 / 2), f_{0}\right\rangle$, не является регулярной $L_{\supset}$-логикой.

(20) $f_{0}(1)=0$ (допущение).

Принимая во внимание утверждения (1) и (20), замечание 9 и соглашение 13 , приходим к выводу, что

(21) $f_{0}=\neg(0,1,1)$, или $f_{0}=\neg(0,1,1 / 2)$, или $f_{0}=\neg(0,1,0)$, или $f_{0}=\neg(0,1 / 2,1)$, или $f_{0}=\neg(0,1 / 2,1 / 2)$, или $f_{0}=\neg(0,1 / 2,0)$, или $f_{0}=\neg(0,0,1)$, или $f_{0}=\neg(0,0,1 / 2)$, или $f_{0}=\neg(0,0,0)$.

Верны следующие утверждения (22)-(30).

(22) Если $f_{0}=\neg(0,1,1)$, то множество всех $L_{\supset \neg}$-формул, общезначимых в $L_{\supset\urcorner^{-}}$ матрице $\left\langle M(1,0,0,1 / 2), f_{0}\right\rangle$, не является регулярной $L_{\supset-}$-логикой.

(23) Если $f_{0}=\neg(0,1,1 / 2)$, то множество всех $L_{\supset \neg}$-формул, общезначимых в $L_{\supset\urcorner^{-}}$

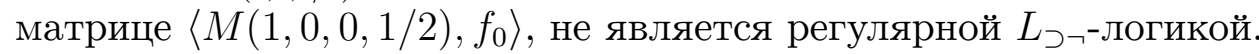

(24) Если $f_{0}=\neg(0,1,0)$, то множество всех $L_{\supset \neg}$-формул, общезначимых в $L_{\supset\urcorner^{-}}$

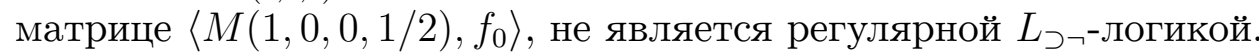

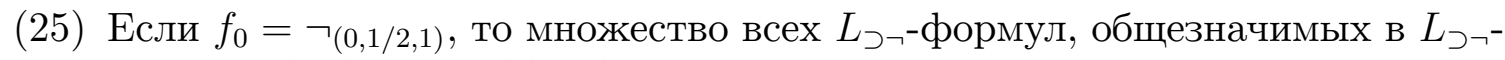

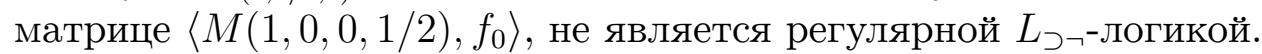

(26) Если $f_{0}=\neg(0,1 / 2,1 / 2)$, то множество всех $L_{\supset-}$-формул, общезначимых в

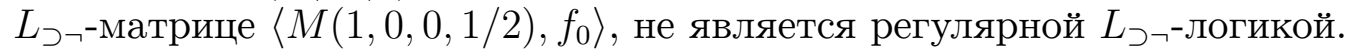

(27) Если $f_{0}=\neg(0,1 / 2,0)$, то множество всех $L_{\supset \neg}$-формул, общезначимых в $L_{\supset\urcorner^{-}}$

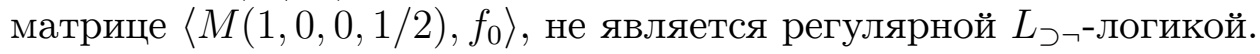




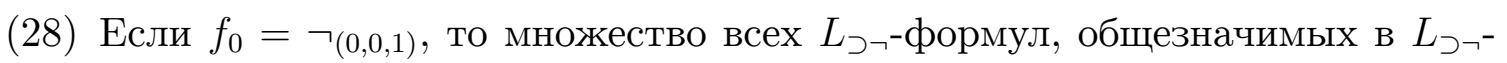

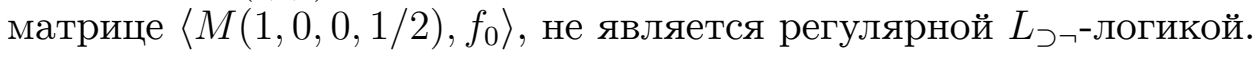

(29) Если $f_{0}=\neg(0,0,1 / 2)$, то множество всех $L_{\supset \neg}$-формул, общезначимых в $L_{\supset\urcorner^{-}}$

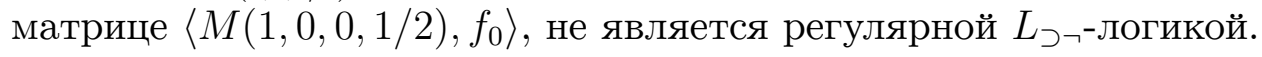

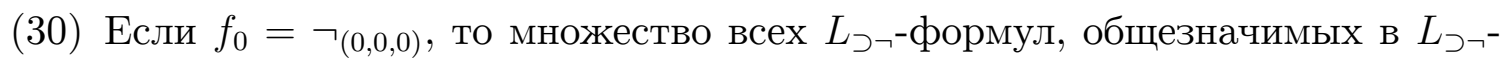

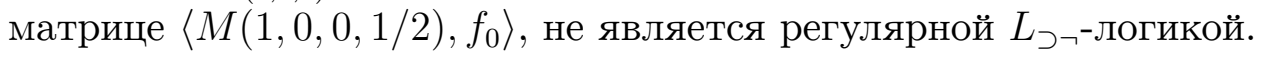

Докажем утверждение (22).

(22.1) $f_{0}=\neg(0,1,1)$ (допущение).

Ввиду утверждения (22.1) и леммы 16 имеем, что

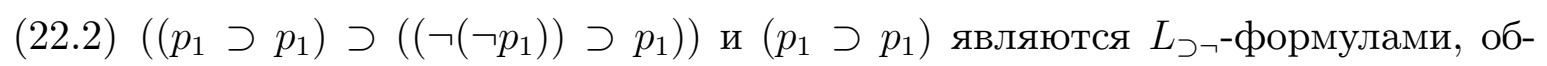

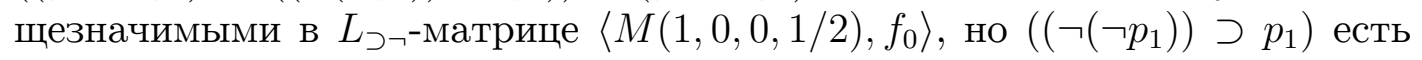
$L_{\supset \neg}$-формула, не являющаяся $L_{\supset \neg}$-формулой, общезначимой в $L_{\supset}$-матрице $\left\langle M(1,0,0,1 / 2), f_{0}\right\rangle$.

Опираясь на утверждение (22.2), получаем, что

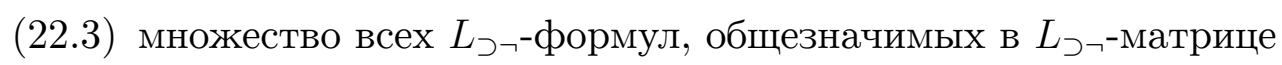

$$
\left\langle M(1,0,0,1 / 2), f_{0}\right\rangle
$$

не замкнуто относительно правила modus ponens в $L_{\supset \neg}$.

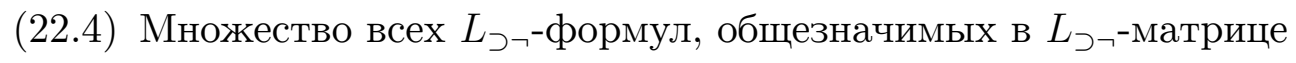

$$
\left\langle M(1,0,0,1 / 2), f_{0}\right\rangle,
$$

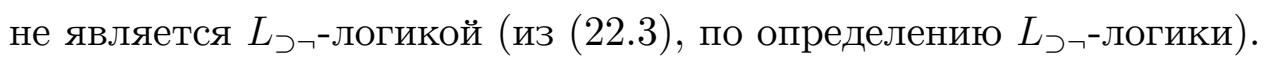

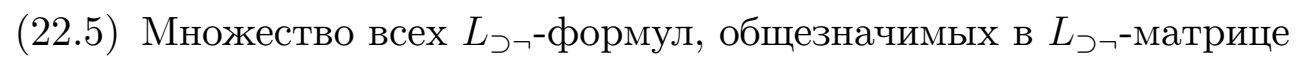

$$
\left\langle M(1,0,0,1 / 2), f_{0}\right\rangle
$$

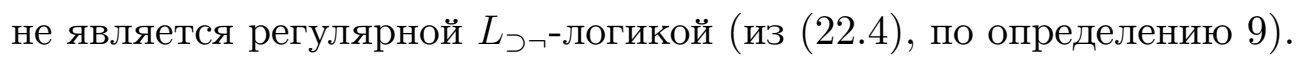

Снимая допущение (22.1), завершаем доказательство утверждения (22).

Утверждение (22) доказано.

Опираясь на леммы 19, 22 и 23, легко построить доказательства утверждений $(25),(28)$ и (29), аналогичные данному выше и опирающемуся на лемму 16 доказательству утверждения (22).

Докажем утверждение (23).

(23.1) $f_{0}=\neg(0,1,1 / 2)$ (допущение).

Ввиду утверждения (23. 1) и леммы 17 имеем, что 
Владимир Попов. К проблеме расширения матричной семантики...

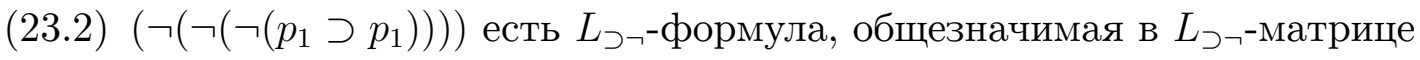

$$
\left\langle M(1,0,0,1 / 2), f_{0}\right\rangle .
$$

Легко убедиться, что

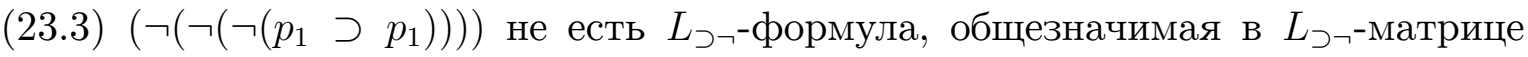
$M\left(C l_{\supset \neg}\right)$.

Опираясь на утверждение (23.3) и на соглашение 11, получаем, что

$$
\left(\neg\left(\neg\left(\neg\left(p_{1} \supset p_{1}\right)\right)\right)\right) \text { не принадлежит множеству } C l_{\supset \neg} \text {. }
$$

В свете утверждений (23.2) и (23.4) ясно, что

(23.5) множество всех $L_{\supset \neg}$-формул, общезначимых в $L_{\supset-}$-матрице

$$
\left\langle M(1,0,0,1 / 2), f_{0}\right\rangle
$$

не включается в $C l_{\supset \neg}$.

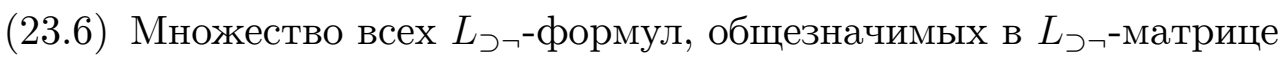

$$
\left\langle M(1,0,0,1 / 2), f_{0}\right\rangle
$$

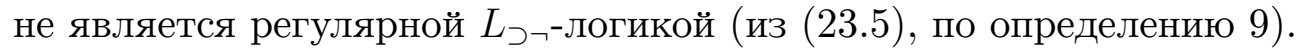

Снимая допущение (23.1), завершаем доказательство утверждения (23).

Утверждение (23) доказано.

Опираясь на леммы 18, 20, 21 и 24, легко построить доказательства утверждений $(24),(26),(27)$ и (30), аналогичные данному выше и опирающемуся на лемму 17 доказательству утверждения (23).

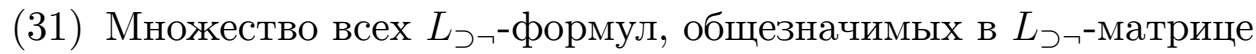

$$
\left\langle M(1,0,0,1 / 2), f_{0}\right\rangle
$$

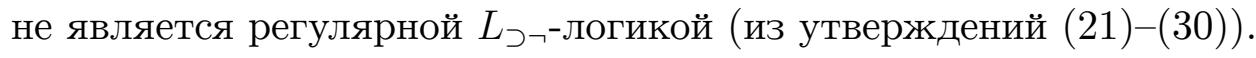

В свете утверждения (31) ясно, что

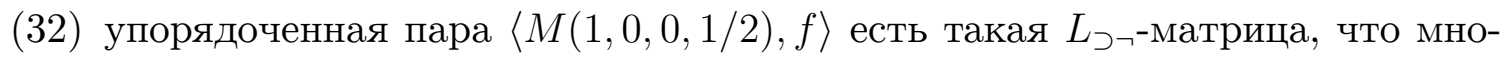

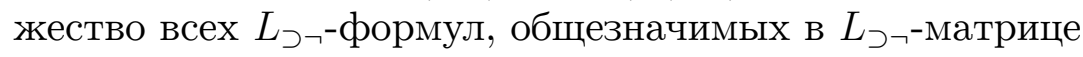

$$
\langle M(1,0,0,1 / 2), f\rangle
$$

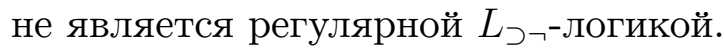


Снимая допущение (1) и обобщая, завершаем доказательство теоремы 2.

Теорема 2 доказана.

Итак, доказано, что не существует такой унарной операции $f$ на множе-

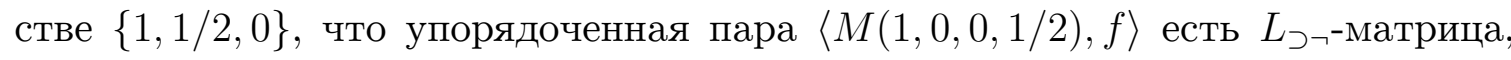

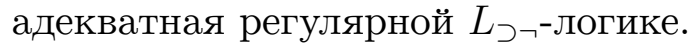

Теперь докажем, что для всякого целого положительного числа $n$ существует $(n+3)$-значная $L_{\supset}$-матрица $K$ с единственным выделенным значением, адекватная классической импликативной логике и удовлетворяющая условию: для всякой унарной операции $f$ на носителе этой $L_{\supset}$-матрицы упорядоченная

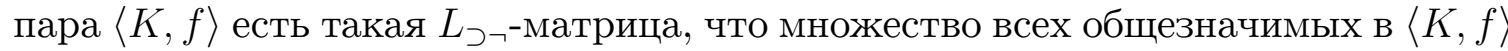
формул не является логикой $C l_{\supset \neg}$.

Соглашение 14. Обозначаем через $\supset\langle n\rangle$ бинарную операцию на $\{1,2, \ldots, n, n+$ $1,1 / 2,0\}$ (п есть целое положительное число), определяемую следующей таблищей

\begin{tabular}{c|ccccccc}
$\supset\langle n\rangle$ & 1 & 2 & $\ldots$ & $n$ & $n+1$ & $1 / 2$ & 0 \\
\hline 1 & 1 & 1 & $\ldots$ & 1 & 1 & 1 & 0 \\
2 & 1 & 1 & $\ldots$ & 1 & 1 & 1 & 0 \\
$\cdot$ & & &. & & & & \\
$\cdot$ & & &. & & & & \\
$\cdot$ & & &. & & & & \\
$n$ & 1 & 1 & $\ldots$ & 1 & 1 & 1 & 0 \\
$n+1$ & 1 & 1 & $\ldots$ & 1 & 1 & 1 & 0 \\
$1 / 2$ & 1 & 1 & $\ldots$ & 1 & 1 & 1 & 0 \\
0 & 1 & 1 & $\ldots$ & 1 & 1 & $1 / 2$ & 1
\end{tabular}

Можно доказать, что для всякого целого положительного числа $n$ существует единственная упорядоченная тройка с первым членом $\{1,2, \ldots, n, n+$ $1,1 / 2,0\}$, со вторым членом $\{1\}$ и с третьим членом $\supset\langle n\rangle$.

Соглашение 15. Упорядоченную тройку $\langle\{1,2, \ldots, n, n+1,1 / 2,0\},\{1\}, \supset\langle n\rangle\rangle$, где $n$ есть иелое положительное число,обозначаем через $M_{\langle n\rangle}$.

Очевидна справедливость следующего замечания 9.

Замечание 10. Для всякого челого положительного числа $n M_{\langle n\rangle}$ есть $L_{\supset \neg-}$ матрица.

Соглашение 16. Определяемое таблищей

$$
\begin{array}{l|llllll}
r & 0 & 1 / 2 & 1 & 2 & 3 & \ldots \\
\hline & 0 & 1 / 2 & 1 & 1 & 1 & \ldots
\end{array}
$$

отображение множества $\{0,1 / 2,1,2,3, \ldots\}$ на множество $\{1,1 / 2,0\}$ обозначаем через $r$. 
Владимир Попов. К проблеме расширения матричной семантики...

Определение 12. Называем r-преобразованием упорядоченной пары, первый член q которой есть пропозичиональная переменная языка $L_{\supset}$ и второй член $x$ которой есть элемент множества $\{0,1 / 2,1,2,3, \ldots\}$, упорядоченную пару, первый член которой есть q и второй член которой есть $r(x)$.

Определение 13. Называем $r$-преобразованием отображения $v$ множества всех пропозиииональных переменных языка $L_{\supset}$ в $\{0,1 / 2,1,2,3, \ldots\}$ множество всех таких упорядоченных пар, каждая из которых есть г-преобразование упорядоченной пары из $v$.

Замечание 11. Доказано, что для всякого отображения множества всех пропозициональных переменных языка $L_{\supset}$ в $\{0,1 / 2,1,2,3, \ldots\}$ существует единственное r-преобразование этого отображения множества всех пропозичиональных переменных языка $L_{\supset}$ в $\{0,1 / 2,1,2,3, \ldots\}$.

Соглашение 17. Обозначаем $r$-преобразование отображения $v$ множества всех пропозиционалъных переменных языка $L_{\supset}$ в $\{0,1 / 2,1,2,3, \ldots\}$ через $r[v]$.

Замечание 12. Для всякого челого положительного числа $n$ и для всякой оценки $v$ языка $L_{\supset}$ в $L_{\supset}$-матрице $M_{\langle n\rangle} r[v]$ есть оценка языка $L_{\supset}$ в $L_{\supset}$-матрице $M(1,0,0,1 / 2)$.

Лемма 25. Для всякой $L_{\supset}$ формулы $A$, для всякого целого положительного

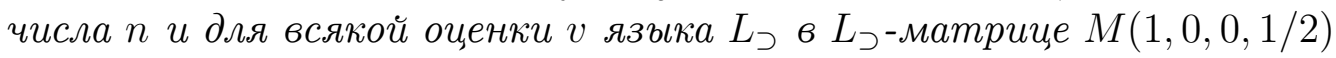

$$
\varphi_{M(1,0,0,1 / 2)}(\langle A, v\rangle)=\varphi_{M_{\langle n\rangle}}(\langle A, v\rangle) .
$$

Доказательство леммы 25 проведено индукцией по построению $L_{\supset}$-формулы.

Лемма 26. Для всякой $L_{\supset}$ бормуль $A$, для всякого иелого положительного

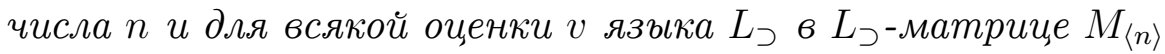

$$
r\left(\varphi_{M_{\langle n\rangle}}(\langle A, v\rangle)\right)=\varphi_{M(1,0,0,1 / 2)}(\langle A, r[v]\rangle) .
$$

Доказательство леммы 26 проведено индукцией по построению $L_{\supset}$-формулы.

В свете табличного определения бинарной операции $\supset_{\langle n\rangle}$ на $\{1,2, \ldots, n, n+$ $1,1 / 2,0\}$ ( $n$ есть целое положительное число) очевидна справедливость следующей леммы 27.

Лемма 27. Для всякой $L_{\supset}$ бормулы $A$, для всякого целого положительного числа $n$ и для всякой оценки $v$ языка $L_{\supset}$ в $L_{\supset}$-матрице $M_{\langle n\rangle}$ : если $A$ не есть пропозициональная переменная языка $L_{\supset}, \operatorname{mo} \varphi_{M_{\langle n\rangle}}(\langle A, v\rangle) \in\{1,1 / 2,0\}$.

Лемма 28. Для всякой $L_{\supset}$ бормулы $A$, для всякого целого положительного числа п: если существует такая ощенка $v$ языка $L_{\supset}$ в $L_{\supset}$-матрище $M_{\langle n\rangle}$, что $\varphi_{M_{\langle n\rangle}}(\langle A, v\rangle) \neq 1$, то существует такая оценка $v$ языка $L_{\supset}$ в $L_{\supset}$-матрище $M_{\langle n\rangle}$, что $\varphi_{M_{\langle n\rangle}}(\langle A, v\rangle)$ не принадлежит множеству $\{1,2,3, \ldots\}$.

Доказательство леммы 28 проведено с использованием леммы 27. 
Лемма 29. Для всякой $L_{\supset}$ формулы $A$ и для всякого иелого положительного

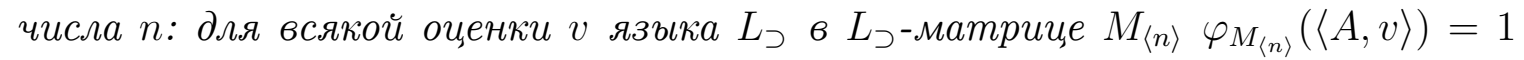
тогда и толъко тогда, когда для всякой оценки $v$ языка $L_{\supset}$ в $L_{\supset}$-матрице $M(1,0,0,1 / 2) \varphi_{M(1,0,0,1 / 2)}(\langle A, v\rangle)=1$.

Доказательство леммы 29 проводим от противного.

(1) Неверна лемма 29 (допущение).

(2) Существует $L_{\supset}$-формула $A$ и существует целое положительное число $n$ : (для всякой оценки $v$ языка $L_{\supset}$ в $L_{\supset}$-матрице $M_{\langle n\rangle} \varphi_{M_{\langle n\rangle}}(\langle A, v\rangle)=1$ и

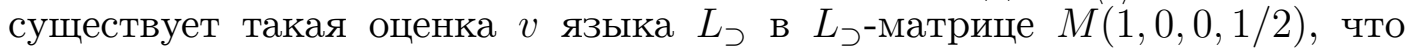
$\varphi_{M(1,0,0,1 / 2)}(\langle A, v\rangle) \neq 1$ ) или (для всякой оценки $v$ языка $L_{\supset}$ в $L_{\supset}$-матрице $M(1,0,0,1 / 2) \varphi_{M(1,0,0,1 / 2)}(\langle A, v\rangle)=1$ и существует такая оценка $v$ языка

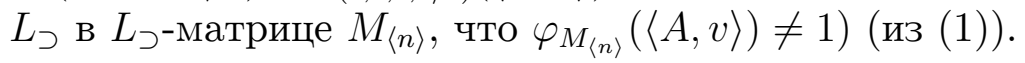

Пусть

(3) $A^{\prime}$ есть $L_{\supset}$-формула, $n^{\prime}$ есть целое положительное число, (для всякой оцен-

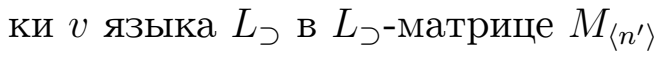

$$
\varphi_{M_{\left\langle n^{\prime}\right\rangle}}\left(\left\langle A^{\prime}, v\right\rangle\right)=1
$$

и существует такая оценка $v$ языка $L_{\supset}$ в $L_{\supset}$-матрице $M(1,0,0,1 / 2)$, что $\varphi_{M(1,0,0,1 / 2)}\left(\left\langle A^{\prime}, v\right\rangle\right) \neq 1$ ) или (для всякой оценки $v$ языка $L_{\supset}$ в $L_{\supset}$-матрице $M(1,0,0,1 / 2)$

$$
\varphi_{M(1,0,0,1 / 2)}\left(\left\langle A^{\prime}, v\right\rangle\right)=1
$$

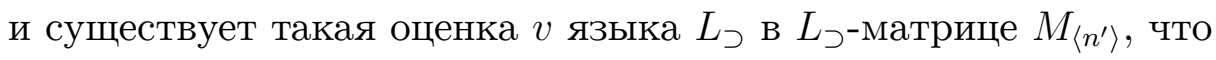

$$
\left.\varphi_{M_{\left\langle n^{\prime}\right\rangle}}\left(\left\langle A^{\prime}, v\right\rangle\right) \neq 1\right)
$$

(4) $A^{\prime}$ есть $L_{\supset}$-формула (из (3)).

(5) $n^{\prime}$ есть целое положительное число (из (3)).

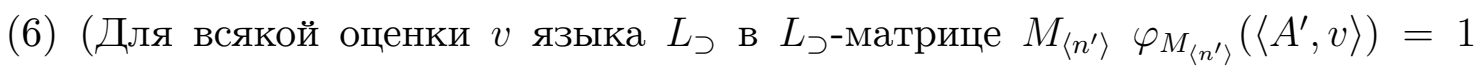

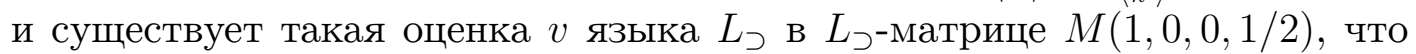

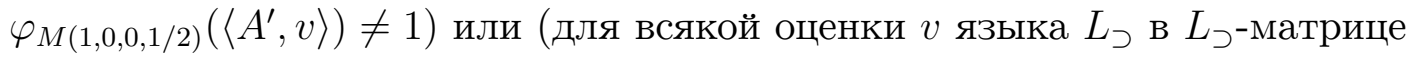
$M(1,0,0,1 / 2) \varphi_{M(1,0,0,1 / 2)}\left(\left\langle A^{\prime}, v\right\rangle\right)=1$ и существует такая оценка $v$ языка

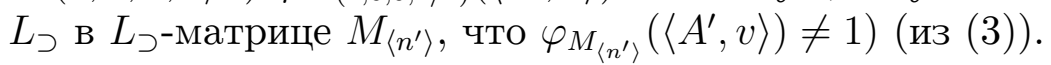

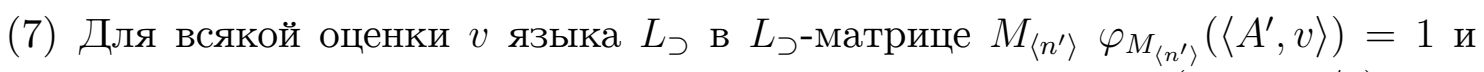
существует такая оценка $v$ языка $L_{\supset}$ в $L_{\supset}$-матрице $M(1,0,0,1 / 2)$, что $\varphi_{M(1,0,0,1 / 2)}\left(\left\langle A^{\prime}, v\right\rangle\right) \neq 1$ (допущение).

(8) Для всякой оценки $v$ языка $L_{\supset}$ в $L_{\supset}$-матрице $M_{\left\langle n^{\prime}\right\rangle} \varphi_{M_{\left\langle n^{\prime}\right\rangle}}\left(\left\langle A^{\prime}, v\right\rangle\right)=1$ (из (7)). 
Владимир Попов. К проблеме расширения матричной семантики...

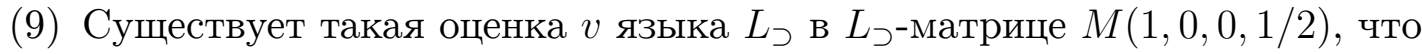

$$
\left.\varphi_{M(1,0,0,1 / 2)}\left(\left\langle A^{\prime}, v\right\rangle\right) \neq 1 \text { (из }(7)\right) .
$$

Пусть

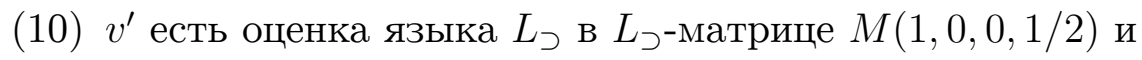

$$
\varphi_{M(1,0,0,1 / 2)}\left(\left\langle A^{\prime}, v^{\prime}\right\rangle\right) \neq 1
$$

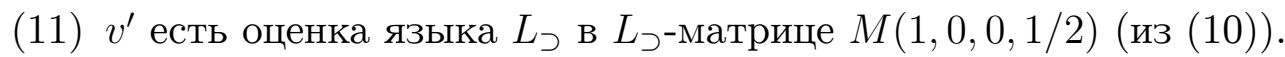

(12) $\varphi_{M(1,0,0,1 / 2)}\left(\left\langle A^{\prime}, v^{\prime}\right\rangle\right) \neq 1$ (из (10)).

(13) $\varphi_{M_{\left\langle n^{\prime}\right\rangle}}\left(\left\langle A^{\prime}, v^{\prime}\right\rangle\right)=\varphi_{M(1,0,0,1 / 2)}\left(\left\langle A^{\prime}, v^{\prime}\right\rangle\right)$ (из (4) и (11), по лемме 25).

(14) $\varphi_{M_{\left\langle n^{\prime}\right\rangle}}\left(\left\langle A^{\prime}, v^{\prime}\right\rangle\right) \neq 1$ (из (12) и $\left.(13)\right)$.

(15) $v^{\prime}$ есть оценка языка $L_{\supset}$ в $L_{\supset}$-матрице $M_{\left\langle n^{\prime}\right\rangle}$ (из $(11)$ и того, что всякая оценка языка $L_{\supset}$ в $L_{\supset}$-матрице $M(1,0,0,1 / 2)$ является оценкой языка $L_{\supset}$

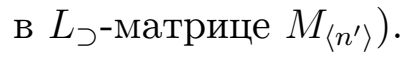

(16) $\varphi_{M_{\left\langle n^{\prime}\right\rangle}}\left(\left\langle A^{\prime}, v^{\prime}\right\rangle\right)=1$ (из (8) и $\left.(15)\right)$.

Утверждение (16) противоречит утверждению (14). Следовательно, неверно допущение (7). Опираясь на утверждение (6) и учитывая, что неверно допущение (7), получаем, что

(17) для всякой оценки $v$ языка $L_{\supset}$ в $L_{\supset}$-матрице $M(1,0,0,1 / 2)$

$$
\varphi_{M(1,0,0,1 / 2)}\left(\left\langle A^{\prime}, v\right\rangle\right)=1
$$

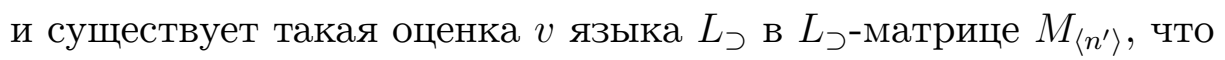

$$
\varphi_{M_{\left\langle n^{\prime}\right\rangle}}\left(\left\langle A^{\prime}, v\right\rangle\right) \neq 1
$$

(18) Для всякой оценки $v$ языка $L_{\supset}$ в $L_{\supset}$-матрице $M(1,0,0,1 / 2)$

$$
\left.\varphi_{M(1,0,0,1 / 2)}\left(\left\langle A^{\prime}, v\right\rangle\right)=1 \text { (из }(17)\right) \text {. }
$$

(19) Существует такая оценка $v$ языка $L_{\supset}$ в $L_{\supset \text {-матрице } M_{\left\langle n^{\prime}\right\rangle} \text {, что }}$

$$
\left.\varphi_{M_{\left\langle n^{\prime}\right\rangle}}\left(\left\langle A^{\prime}, v\right\rangle\right) \neq 1 \text { (из }(17)\right) \text {. }
$$

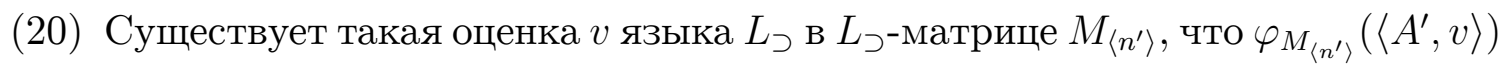
не принадлежит множеству $\{1,2,3, \ldots\}$ (из (4), (5), (19), по лемме 28).

Пусть 


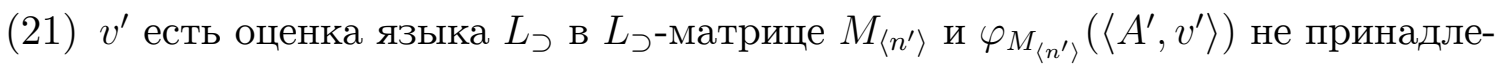
жит множеству $\{1,2,3, \ldots\}$.

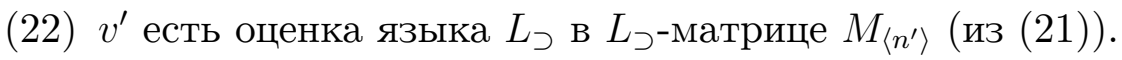

(23) $\varphi_{M_{\left\langle n^{\prime}\right\rangle}}\left(\left\langle A^{\prime}, v^{\prime}\right\rangle\right)$ не принадлежит множеству $\{1,2,3, \ldots\}$ (из $\left.(21)\right)$.

(24) $r\left(\varphi_{M_{\left\langle n^{\prime}\right\rangle}}\left(\left\langle A^{\prime}, v^{\prime}\right\rangle\right)\right)=\varphi_{M(1,0,0,1 / 2)}\left(\left\langle A^{\prime}, r\left(v^{\prime}\right)\right\rangle\right)$ (из (4), (5), (22), по лемме 26).

Ясно, что

(25) $\varphi_{M_{\left\langle n^{\prime}\right\rangle}}\left(\left\langle A^{\prime}, v^{\prime}\right\rangle\right) \in\{0,1 / 2,1,2,3, \ldots\}$.

(26) $\varphi_{M_{\left\langle n^{\prime}\right\rangle}}\left(\left\langle A^{\prime}, v^{\prime}\right\rangle\right) \in\{0,1 / 2\}$ (из $(23)$ и $\left.(25)\right)$.

Опираясь на утверждение (26) и на соглашение 16, получаем, что

(27) $r\left(\varphi_{M_{\left\langle n^{\prime}\right\rangle}}\left(\left\langle A^{\prime}, v^{\prime}\right\rangle\right)\right) \neq 1$.

(28) $\varphi_{M(1,0,0,1 / 2)}\left(\left\langle A^{\prime}, r\left(v^{\prime}\right)\right\rangle\right) \neq 1$ (из $(24)$ и $\left.(27)\right)$.

(29) $r\left[v^{\prime}\right]$ есть оценка языка $L_{\supset}$ в $L_{\supset}$-матрице $M(1,0,0,1 / 2)$ (из $(22)$, по замечанию 12).

(30) $\varphi_{M(1,0,0,1 / 2)}\left(<\left\langle A^{\prime}, r\left(v^{\prime}\right)\right\rangle\right)=1$ (из (19) и $\left.(29)\right)$.

Утверждение (30) противоречит утверждению (28). Следовательно, неверно допущение (1).

Лемма 29 доказана.

Опираясь на теорему 1 , лемму 29 , определение 5 и замечание 9 , делаем вывод о том, что верна следующая теорема 3.

Теорема 3. Для всякой $L_{\supset}$-бормулы $A$ и для всякого иелого положительного числа $n: A \in C l_{\supset}$ тогда и только тогда, когда $A$ есть $L_{\supset}$-формула, общезначимая в $L_{\supset}$-матрице $M_{\langle n\rangle}$.

Теорема 4. Для всякого целого положительного числа $n$ и для всякой унарной операчии $f$ на множестве $\{1,2, \ldots, n, n+1,1 / 2,0\}$ упорядоченная пара $\left\langle M_{\langle n\rangle}, f\right\rangle$

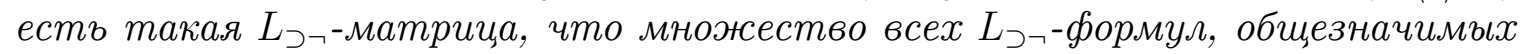

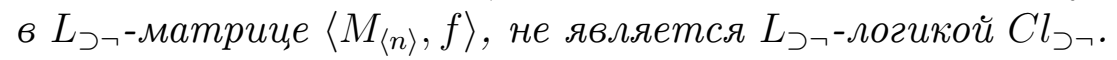

Докажем теорему 4.

(1) $m$ есть целое положительное число (допущение).

(2) $f^{\prime}$ есть унарная операция на множестве $\{1,2, \ldots, n, n+1,1 / 2,0\}$ (допущение).

Очевидно, что

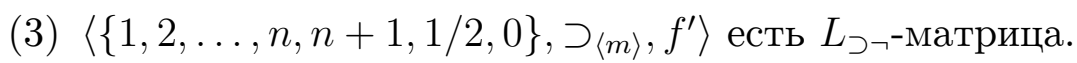


Владимир Попов. К проблеме расширения матричной семантики...

Опираясь на утверждение (3) и на тот факт, что

$$
\left\langle\{1,2, \ldots, n, n+1,1 / 2,0\}, \supset_{\langle m\rangle}, f^{\prime}\right\rangle=\left\langle\left\langle\{1,2, \ldots, n, n+1,1 / 2,0\}, \supset_{\langle m\rangle}\right\rangle, f^{\prime}\right\rangle,
$$

получаем, что

(4) $\left\langle\left\langle\{1,2, \ldots, n, n+1,1 / 2,0\}, \supset_{\langle m\rangle}\right\rangle, f^{\prime}\right\rangle$ есть $L_{\supset-}$-матрица.

(5) $\left\langle M_{\langle m\rangle}, f^{\prime}\right\rangle$ есть $L_{\supset-}$-матрица (из (4), по соглашению 15).

Принимая во внимание утверждение (2), приходим к выводу, что

(6) $f^{\prime}(1) \in\{1,2, \ldots, m, m+1\}$ или $f^{\prime}(1) \in\{1 / 2,0\}$.

Легко проверить, что верны следующие утверждения (7), (8), (9) и (10).

(7) Если $f^{\prime}(1) \in\{1,2, \ldots, m, m+1\}$, то $\left(\left(p_{1} \supset p_{1}\right) \supset\left(\neg\left(p_{1} \supset p_{1}\right)\right)\right)$ есть $L_{\supset \neg^{-}}$

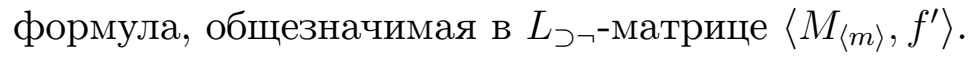

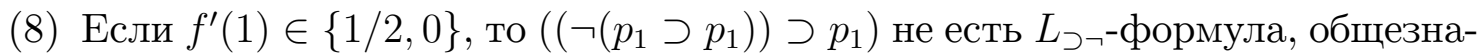

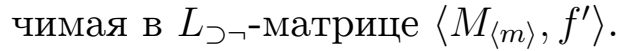

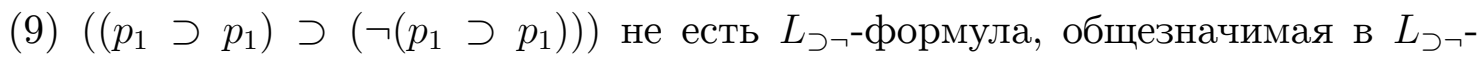
матрице $M\left(C l_{\supset \neg}\right)$.

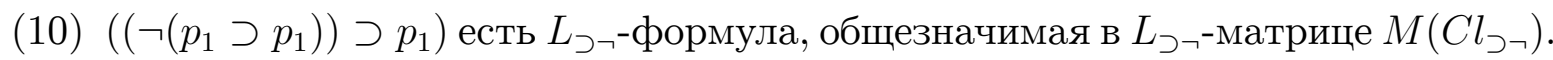

(11) $\left(\left(p_{1} \supset p_{1}\right) \supset\left(\neg\left(p_{1} \supset p_{1}\right)\right)\right)$ не принадлежит множеству $C l_{\supset \neg}$ (из $(9)$, по соглашению 11).

(12) $\left(\left(\neg\left(p_{1} \supset p_{1}\right)\right) \supset p_{1}\right)$ принадлежит множеству $C l_{\supset \neg}$ (из $(10)$, по соглашению $11)$.

В свете утверждений (7) и (11) ясно, что

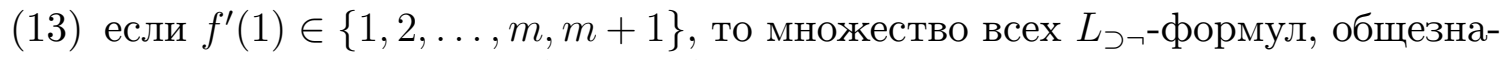

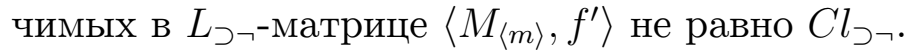

В свете утверждений (8) и (12) ясно, что

(14) если $f^{\prime}(1) \in\{1 / 2,0\}$, то множество всех $L_{\supset \neg}$-формул, общезначимых в

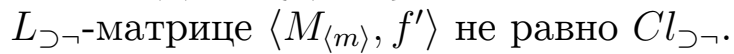

Опираясь на утверждения (6), (13), (14) и на замечание 5, получаем, что

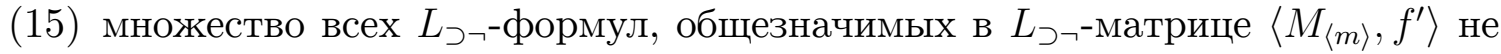

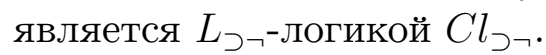

Итак,

(16) $\left\langle M_{\langle m\rangle}, f^{\prime}\right\rangle$ есть такая $L_{\supset\urcorner}$-матрица, что множество всех $L_{\supset\urcorner}$-формул, об-

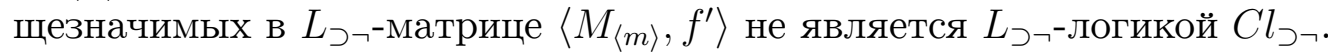


Снимая допущения (2) и (4) и обобщая, завершаем доказательство теоремы 4.

Теорема 4 доказана.

Итак, найдена трехзначная логическая матрица $M(1,0,0,1 / 2)$ с одним выделенным значением, которая адекватна классической импликативной логике, но которая не может быть расширена до логической матрицы, адекватной классической импликативно-негативной логике (не может быть расширена в том смысле, что не существует такой унарной операции $f$, что упорядоченная пара $\langle M(1,0,0,1 / 2), f\rangle$ является логической матрицей, адекватной классической импликативно-негативной логике). Более того, не существует такой унарной операции $f$, что упорядоченная пара $\langle M(1,0,0,1 / 2), f\rangle$ является логической матрицей, адекватной регулярной $L_{\supset}$-логике. Доказано также, что для всякого целого положительного числа $n$ существует такая $(n+3)$-значная логическая матрица $K$ с одним выделенным значением, адекватная классической импликативной логике, что для всякой унарной операции $f$ на носителе этой логической матрицы упорядоченная пара $\langle K, f\rangle$ есть логическая матрица, не являющаяся логической матрицей, адекватной классической импликативно-негативной логике. Завершаем статью анонсом двух следующих результатов: (i) всякая адекватная классической импликативной логике трехзначная логическая матрица $K$ с единственным выделенным значением, для которой не существует такой унарной операции $f$, что $\langle K, f\rangle$ является логической матрицей, адекватной классической импликативно-негативной логике, изоморфна логической матрице $M(1,0,0,1 / 2)$, (ii) $M(1 / 2,1,0,1 / 2)$ есть единственная логическая матрица, которая отлична от $M(1,0,0,1 / 2)$ и выполняет условия: (а) носитель логической матрицы $M(1 / 2,1,0,1 / 2)$ есть $\{1,1 / 2,0\}$, (б) выделенное множество логической матрицы $M(1 / 2,1,0,1 / 2)$ есть $\{1\}$, (в) логическая матрица $M(1 / 2,1,0,1 / 2)$ адекватна классической импликативной логике, (г) не существует такой унарной операции $f$, что упорядоченная пара $\langle M(1 / 2,1,0,1 / 2), f\rangle$ является логической матрицей, адекватной классической импликативно-негативной логике.

\section{Литература}

Соболев 1979 - Соболев С. К. Импликативное пропозициональное исчисление // Математическая энциклопедия : Гл. ред. И. М. Виноградов. Т. 2: Д - Коо. М.: Советская энциклопедия, 1979. С. 523.

Черч 1960 - Черч А. Введение в математическую логику. Т. 1. М.: Издательство иностранной литературы, 1960. 